\title{
Zitterbewegung (Trembling Motion) of Electrons in Graphene
}

\author{
Tomasz M. Rusin ${ }^{1}$ and Wlodek Zawadzki ${ }^{2}$ \\ ${ }^{1}$ Orange Customer Service sp. z o. o., Warsaw \\ ${ }^{2}$ Institute of Physics, Polish Academy of Sciences, Al. Lotników, Warsaw \\ Poland
}

\section{Introduction}

This chapter describes a somewhat mysterious phenomenon known in the literature under the German name of "Zitterbewegung" (trembling motion). Both the phenomenon and its name were conceived by Erwin Schrodinger who, in 1930, published the paper Ueber die kraeftefreie Bewegung in der relativistischen Quantenmechanik in which he observed that in the Dirac equation, describing relativistic electrons in a vacuum, the $4 \times 4$ operators corresponding to components of relativistic velocity do not commute with the free-electron Hamiltonian (Barut \& Bracken, 1981; Schroedinger, 1930). In consequence, the electron velocity is not a constant of the motion also in absence of external fields. Such an effect in a vacuum must be of a quantum nature as it does not obey Newton's first law of classical motion. Schrodinger calculated the resulting time dependence of the electron velocity and position concluding that, in addition to classical motion, they experience very fast periodic oscillations which he called Zitterbewegung (ZB). Schrodinger's idea stimulated numerous theoretical investigations but no experiments since the predicted frequency $\hbar \omega_{Z} \simeq 2 m_{0} c^{2} \simeq 1 \mathrm{MeV}$ and the amplitude of about $\lambda_{c}=\hbar / m c \simeq 3.86 \times 10^{-3} \AA$ are not accessible to current experimental techniques. It was recognized that the $\mathrm{ZB}$ is due to an interference of states corresponding to the positive and negative electron energies resulting from the Dirac equation (Bjorken \& Drell, 1964; Greiner, 1994; Sakurai, 1997). Lock (1979) showed that, if an electron is represented by a wave packet, its ZB has a transient character, i.e. it disappears with time.

It was conceived years later that the trembling electron motion should occur also in crystalline solids if their band structure could be represented by a two-band model reminiscent of the Dirac equation (Cannata et al., 1990; Lurie \& Cremer, 1970; Vonsovskii et al., 1990; Zawadzki, 1997). An intense interest in ZB of electrons in semiconductors was launched only in 2005 (Schliemann et al., 2005; Zawadzki, 2005). There followed a wave of theoretical considerations for various semiconductor and other systems. It was recognized that the phenomenon of $\mathrm{ZB}$ occurs every time one deals with two or more interacting energy bands (Cserti \& David, 2006; Rusin \& Zawadzki, 2007a; Winkler et al., 2007).

As mentioned above, in a vacuum the $\mathrm{ZB}$ characteristics are not favorable. In solids, the $\mathrm{ZB}$ characteristics are much better but it is difficult to observe the motion of a single electron. However, recently Gerritsma et al. (2010) simulated experimentally the Dirac equation and 
the resulting electron Zitterbewegung with the use of trapped ions and laser excitations. The power of the simulation method is that one can adjust experimentally the essential parameters of the Dirac equation: $m c^{2}$ and $c$, and thus achieve more favorable values of the $\mathrm{ZB}$ frequency and amplitude. Interestingly, it turned out that analogues of ZB can occur also in classical wave propagation phenomena. Several predictions were made, but in two systems, namely macroscopic sonic crystals (Zhang \& Liu, 2008), and photonic superlattices (Dreisow et al., 2010), the ZB-like effects were actually observed.

\section{ZB of electrons in graphene}

Here we consider the $\mathrm{ZB}$ in three modern materials: monolayer graphene, bilayer graphene and carbon nanotubes. Monolayer graphene is currently by far the most studied material in the world (Novoselov et al., 2004). The description is based mostly on our theoretical works (Rusin \& Zawadzki, 2007b; 2008; 2009), but papers of other authors concerned with $\mathrm{ZB}$ in graphene are also mentioned. It is emphasized that the trembling electrons should emit electromagnetic radiation which would provide a direct evidence for the existence of this phenomenon. We discuss the origin of ZB in crystalline solids not going into detailed mathematical derivations. An exact formulation of the electron dynamics in monolayer graphene in the presence of a magnetic field is presented in Appendix A. The electron classical velocity and its mass for a linear energy band of monolayer graphene are considered in Appendix B. It is hoped that our presentation will simulate experimental investigations of the electron trembling motion in graphene.

\subsection{Bilayer graphene}

We begin our description considering bilayer graphene since the results can be obtained in the analytical form, which allows one to see directly important features of the trembling motion. Two-dimensional Hamiltonian for bilayer graphene in the $\mathrm{K}$ point of Brillouin zone is (McCann \& Fal'ko, 2006)

$$
\hat{H}_{B}=-\frac{1}{2 m^{*}}\left(\begin{array}{cc}
0 & \left(\hat{p}_{x}-i \hat{p}_{y}\right)^{2} \\
\left(\hat{p}_{x}+i \hat{p}_{y}\right)^{2} & 0
\end{array}\right),
$$

where $m^{*}=0.054 m_{0}$. The energy spectrum is $\mathcal{E}_{k}= \pm E_{k}$, where $E_{k}=\hbar^{2} k^{2} / 2 m^{*}$, i.e. there is no energy gap between the parabolic conduction and valence bands. The position operator in the Heisenberg picture is a $2 \times 2$ matrix $\hat{x}(t)=\exp \left(i \hat{H}_{B} t / \hbar\right) \hat{x} \exp \left(-i \hat{H}_{B} t / \hbar\right)$. One calculates

$$
x_{11}(t)=x(0)+\frac{k_{y}}{k^{2}}\left[1-\cos \left(\frac{\hbar k^{2} t}{m^{*}}\right)\right],
$$

where $k^{2}=k_{x}^{2}+k_{y}^{2}$. The third term represents the Zitterbewegung with the frequency $\hbar \omega_{Z}=2 \hbar^{2} k^{2} / 2 m^{*}$, corresponding to the energy difference between the upper and lower energy branches for a given value of $k$. We want to calculate the $\mathrm{ZB}$ of a charge carrier represented by a two-dimensional wave packet

$$
\psi(\boldsymbol{r}, 0)=\frac{1}{2 \pi} \frac{d}{\sqrt{\pi}} \int d^{2} \boldsymbol{k} e^{-\frac{1}{2} d^{2} k_{x}^{2}-\frac{1}{2} d^{2}\left(k_{y}-k_{0 y}\right)^{2}} e^{i \boldsymbol{k} \boldsymbol{r}}\left(\begin{array}{l}
1 \\
0
\end{array}\right) .
$$


The packet is centered at $\boldsymbol{k}_{0}=\left(0, k_{0 y}\right)$ and is characterized by a width $d$. The unit vector $(1,0)$ is a convenient choice, selecting the (11) component of $\hat{x}(t)$, see Eq. (2). An average of $\hat{x}_{11}(t)$ is a two-dimensional integral which can be calculated analytically in the form

$$
\bar{x}_{11}(t)=\langle\psi(\boldsymbol{r})|\hat{x}(t)| \psi(\boldsymbol{r})\rangle=\bar{x}_{c}+\bar{x}_{Z}(t)
$$

where $\bar{x}_{c}=\left(1 / k_{0 y}\right)\left[1-\exp \left(-d^{2} k_{0 y}^{2}\right)\right]$, and

$$
\begin{array}{r}
\bar{x}_{Z}(t)=\frac{1}{k_{0 y}}\left[\exp \left(-\frac{\delta^{4} d^{2} k_{0 y}^{2}}{d^{4}+\delta^{4}}\right) \cos \left(\frac{\delta^{2} d^{4} k_{0 y}^{2}}{d^{4}+\delta^{4}}\right)\right. \\
\left.-\exp \left(-d^{2} k_{0 y}^{2}\right)\right]
\end{array}
$$

in which $\delta=\sqrt{\hbar t / m^{*}}$ contains the time dependence. In Figure 1a we show the ZB of the electron position $\bar{x}_{11}$ as given in Eqs. (4) and (5).

Here are the main features of $\mathrm{ZB}$ following from Eqs. (4) and (5). First, in order to have $\mathrm{ZB}$ in the direction $x$ one needs an initial transverse momentum $\hbar k_{0 y}$. Second, the $\mathrm{ZB}$ frequency depends only weakly on the packet width: $\omega_{Z}=\left(\hbar k_{0 y}^{2} / m^{*}\right)\left(d^{4} /\left(d^{4}+\delta^{4}\right)\right)$, while its amplitude is strongly dependent on the width $d$. Third, the ZB has a transient character since it is attenuated by the exponential term. For small $t$ the amplitude of $\bar{x}_{Z}(t)$ diminishes as $\exp \left(-\Gamma_{Z}^{2} t^{2}\right)$ with

$$
\Gamma_{Z}=\frac{\hbar k_{0 y}}{m^{*} d}
$$

Fourth, as $t$ (or $\delta$ ) increases, the cosine term tends to unity and the first term in Eq. (5) cancels out with the second term. After the oscillations disappear, the charge carrier is displaced by the amount $\bar{x}_{c}$, which is a "remnant" of ZB. Fifth, for very wide packets $(d \rightarrow \infty)$ the exponent in Eq. (5) tends to unity, the oscillatory term is $\cos \left(\delta^{2} k_{0 y}^{2}\right)$ and the last term vanishes. In this limit one recovers undamped ZB oscillations.

Next, we consider other quantities related to $Z B$, beginning by the current. The latter is given by the velocity multiplied by charge. The velocity is simply $\bar{v}_{x}=\partial \bar{x}_{Z} / \partial t$, where $\bar{x}_{Z}$ is given by Eq. (5). The calculated current is plotted in Figure $1 b$, its oscillations are a direct manifestation of $\mathrm{ZB}$. The transient character of $\mathrm{ZB}$ is accompanied by a temporal spreading of the wave packet. The question arises whether the attenuation of $Z B$ is not simply caused by the spreading of the packet. The calculated packet width $\Delta R$ is plotted versus time in Figure 1c. It is seen that during the initial 80 femtoseconds the packet's width increases only twice compared to its initial value, while the ZB disappears almost completely. We conclude that the spreading of the packet is not the main cause of the transient character of ZB. Looking for physical reasons behind the transient character of ZB we decompose the total wave function $\psi(\boldsymbol{r}, t)$ into the positive $(p)$ and negative $(n)$ components $\psi^{p}(\boldsymbol{r}, t)$ and $\psi^{n}(\boldsymbol{r}, t)$. We have

$$
\begin{aligned}
|\psi(t)\rangle & =e^{-i \hat{H} t / \hbar}|\psi(0)\rangle \\
& =e^{-i E t / \hbar}\langle p \mid \psi(0)\rangle|p\rangle+e^{i E t / \hbar}\langle n \mid \psi(0)\rangle|n\rangle,
\end{aligned}
$$




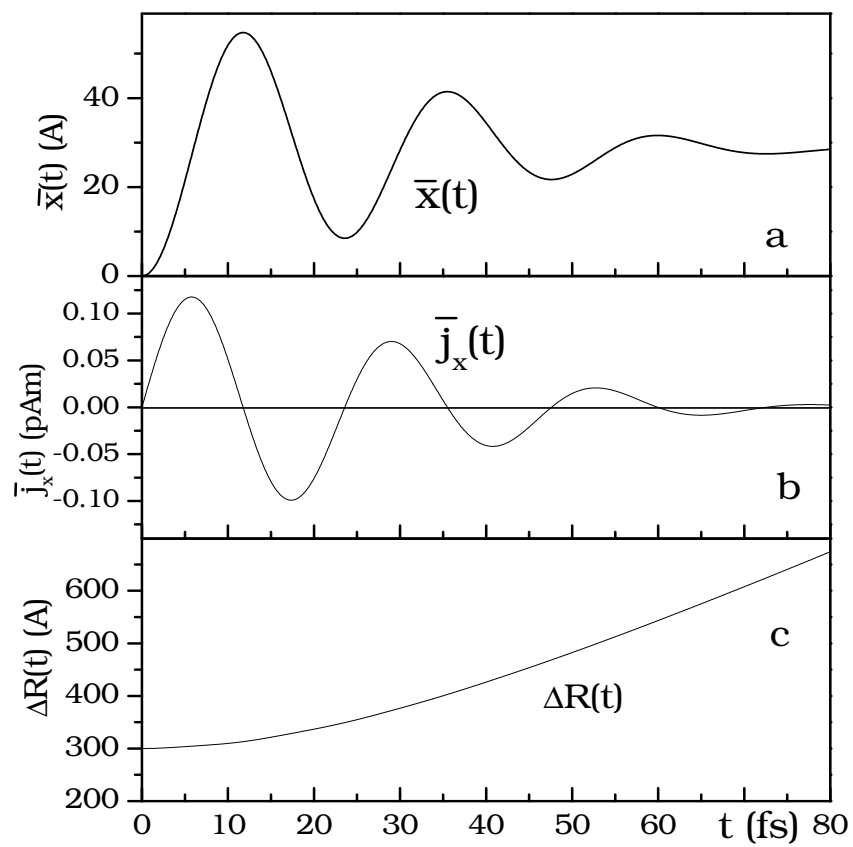

Fig. 1. Zitterbewegung of a charge carrier in bilayer graphene versus time, calculated for a gaussian wave packet width $d=300 \AA$ and $k_{0 y}=3.5 \times 10^{8} \mathrm{~m}^{-1}$ : a) position, b) electric current, $\mathrm{c}$ ) dispersion $\Delta R(t)$. After the $\mathrm{ZB}$ disappears a constant shift of the position remains. After Rusin \& Zawadzki (2007b).

where $|p\rangle$ and $|n\rangle$ are the eigen-functions of the Hamiltonian (1) in $\boldsymbol{k}$ space corresponding to positive and negative energies, respectively. Further

$$
\begin{aligned}
& \langle\boldsymbol{k} \mid p\rangle=\frac{1}{\sqrt{2}}\left(\begin{array}{c}
1 \\
k_{+}^{2} / k^{2}
\end{array}\right) \delta\left(\boldsymbol{k}-\boldsymbol{k}^{\prime}\right), \\
& \langle\boldsymbol{k} \mid n\rangle=\frac{1}{\sqrt{2}}\left(\begin{array}{c}
1 \\
-k_{+}^{2} / k^{2}
\end{array}\right) \delta\left(\boldsymbol{k}-\boldsymbol{k}^{\prime}\right) .
\end{aligned}
$$

After some manipulations we obtain

$$
\begin{array}{r}
\psi^{p}(\boldsymbol{r}, t)=\frac{1}{4 \pi} \frac{d}{\sqrt{\pi}} \int d^{2} \boldsymbol{k} e^{-\frac{1}{2} d^{2}\left(k_{x}^{2}+\left(k_{y}-k_{0 y}\right)^{2}\right)} e^{i \boldsymbol{k} \boldsymbol{r}} e^{-i E t / \hbar} \times \\
\left(\begin{array}{c}
1 \\
k_{+}^{2} / k^{2}
\end{array}\right) .
\end{array}
$$

The function $\psi^{n}(\boldsymbol{r}, t)$ is given by the identical expression with the changed signs in front of $E$ and $k_{+}^{2} / k^{2}$ terms. There is $\psi(\boldsymbol{r}, t)=\psi^{p}(\boldsymbol{r}, t)+\psi^{n}(\boldsymbol{r}, t)$ and $\left\langle\psi^{n} \mid \psi^{p}\right\rangle=0$. Now, one can calculate the average values of $\bar{x}$ and $\bar{y}$ using the positive and negative components in the above sense. We have

$$
\bar{x}(t)=\int\left(\psi^{n}+\psi^{p}\right)^{\dagger} x\left(\psi^{n}+\psi^{p}\right) d^{2} \boldsymbol{r},
$$


so that one deals with four integrals. A direct calculation gives

$$
\begin{gathered}
\int\left|\psi^{p}\right|^{2} x d^{2} \boldsymbol{r}+\int\left|\psi^{n}\right|^{2} x d^{2} \boldsymbol{r}=\bar{x}_{c}, \\
\int \psi^{n \dagger} x \psi^{p} d^{2} \boldsymbol{r}+\int \psi^{p \dagger} x \psi^{n} d^{2} \boldsymbol{r}=\bar{x}_{Z}(t),
\end{gathered}
$$

where $\bar{x}_{c}$ and $\bar{x}_{Z}(t)$ have been defined in Eq. (4). Thus the integrals involving only the positive and only the negative components give the constant shift due to $Z B$, while the mixed terms lead to the ZB oscillations. All terms together reconstruct the result from Eq. (4). Next we calculate the average value $\bar{y}$. There is no symmetry between $\bar{x}$ and $\bar{y}$ because the wave packet is centered around $k_{x}=0$ and $k_{y}=k_{0 y}$. The average value $\bar{y}$ is again given by four integrals. However, now the mixed terms vanish, while the integrals involving the positive and negative components give

$$
\begin{aligned}
\int\left|\psi^{p}\right|^{2} y d^{2} \boldsymbol{r} & =\frac{\hbar k_{0 y}}{2 m^{*}} t, \\
\int\left|\psi^{n}\right|^{2} y d^{2} \boldsymbol{r} & =-\frac{\hbar k_{0 y}}{2 m^{*}} t .
\end{aligned}
$$

This means that the "positive" and "negative" subpackets move in the opposite directions with the velocity $v=\hbar k_{0 y} t / 2 m^{*}$. The relative velocity is $v^{r e l}=\hbar k_{0 y} t / m^{*}$. Each of these packets has the initial width $d$ and it (slowly) spreads in time. After the time $\Gamma_{Z}^{-1}=d / v^{r e l}$ the distance between the two packets equals $d$, so the integrals in Eq. (12) are small, resulting in the diminishing Zitterbewegung amplitude. This reasoning gives the decay constant $\Gamma_{Z}=$ $\hbar k_{0 y} / m^{*} d$, which is exactly what we determined above from the analytical results (see Eq. (6)). Thus, the transient character of the $Z B$ oscillations is due to the increasing spatial separation of the subpackets corresponding to the positive and negative energy states. This confirms our previous conclusion that it is not the packet's slow spreading that is responsible for the attenuation. The separation of subpackets with the resulting decay of ZB turns out to be a general feature of this phenomenon.

\subsection{Monolayer graphene}

Now we consider monolayer graphene. The two-dimensional band Hamiltonian describing its band structure at the K point of the Brillouin zone is (Semenoff, 1984; Slonczewski \& Weiss, 1958; Wallace, 1947)

$$
\hat{H}_{M}=u\left(\begin{array}{cc}
0 & \hat{p}_{x}-i \hat{p}_{y} \\
\hat{p}_{x}+i \hat{p}_{y} & 0
\end{array}\right)
$$

where $u \approx 1 \times 10^{8} \mathrm{~cm} / \mathrm{s}$. The resulting energy dispersion is linear in momentum: $\mathcal{E}= \pm u \hbar k$, where $k=\sqrt{k_{x}^{2}+k_{y}^{2}}$. The quantum velocity in the Schrodinger picture is $\hat{v}_{i}=\partial H_{M} / \partial \hat{p}_{i}$, it does not commute with the Hamiltonian (16). In the Heisenberg picture we have $\hat{\boldsymbol{v}}(t)=$ $\exp \left(i \hat{H}_{M} t / \hbar\right) \hat{v} \exp \left(-i \hat{H}_{M} t / \hbar\right)$. Using Eq. (16) one obtains

$$
v_{x}^{(11)}=u \frac{k_{y}}{k} \sin (2 u k t)
$$




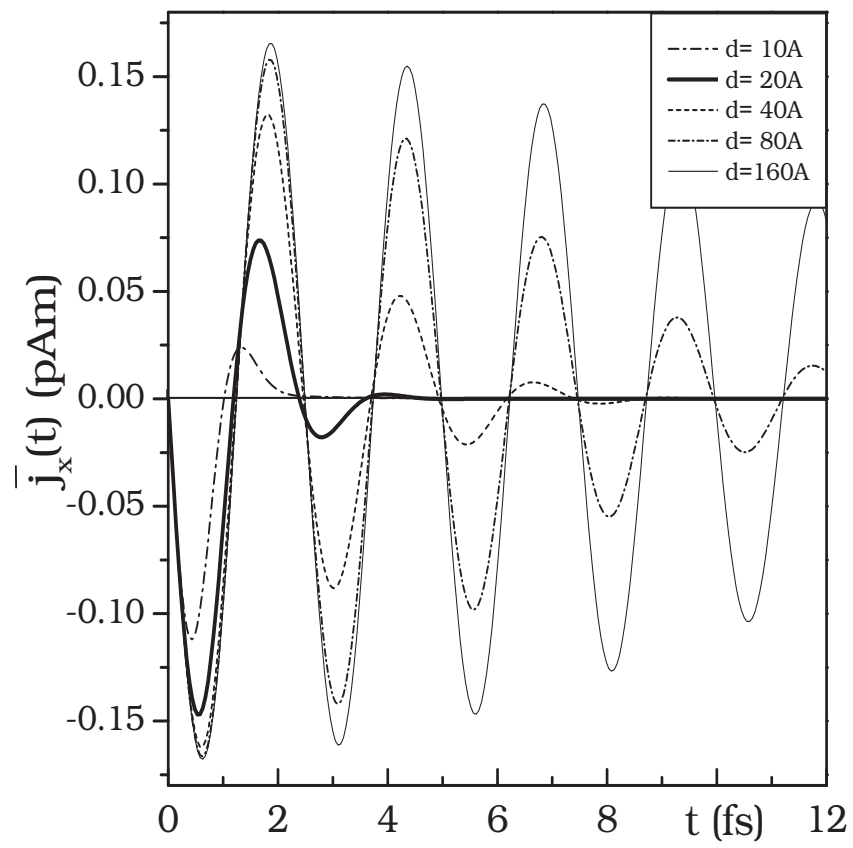

Fig. 2. Oscillatory electric current in the $x$ direction caused by the ZB in monolayer graphene versus time, calculated for a gaussian wave packet with $k_{0 y}=1.2 \times 10^{9} \mathrm{~m}^{-1}$ and various packet widths $d$. Transient character of ZB is clearly seen. After Rusin \& Zawadzki (2007b).

The above equation describes the trembling motion with the frequency $\omega_{Z}=2 u k$, determined by the energy difference between the upper and lower energy branches for a given value of $k$. As before, ZB in the direction $x$ occurs only if there is a non-vanishing momentum $\hbar k_{y}$. One calculates an average velocity (or current) taken over a two-dimensional wave packet with nonzero initial momentum $k_{0 x}$. The results for the current $\bar{j}_{x}=e \bar{v}_{x}$ are plotted in Figure 2 for different realistic packet widths $d$. It is seen that the ZB frequency does not depend on $d$ and is nearly equal to $\omega_{Z}$ given above for the plane wave. On the other hand, the amplitude of $Z B$ depends on $d$ and we deal with decay times of the order of femtoseconds. For small $d$ there are almost no oscillations, for very large $d$ the ZB oscillations are undamped. These conclusions agree with the analytical results for bilayer graphene. The behavior of ZB depends quite critically on the values of $k_{0 y}$ and $d$, which is reminiscent of the damped harmonic oscillator. In the limit $d \rightarrow \infty$ the above results for the electric current resemble those of Katsnelson (2006) for ZB in graphene obtained with the use of plane wave representation.

Maksimova et al. (2008) investigated dynamics of wave packets in monolayer graphene for different pseudo-spin polarizations with the resulting ZB. For specific packet components

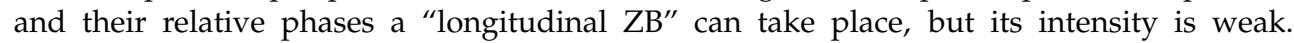
Martinez et al. (2010) considered a creation of electron-hole pairs by a constant electric field in the plane of a monolayer graphene sheet. They showed that, as the pairs undergo the ZB in opposite directions, a Hall-like separation of the charge occurs giving a measurable dipole moment. We note that it is not the time-dependent motion but the ZB shift at large times which is responsible for the charge separation, see Eq. (2) and Figure 1. Englman \& 
Vertesi (2008) calculated a ZB-related electron current in monolayer graphene in the adiabatic approximation and related it to the Berry phase.

\subsection{Carbon nanotubes}

Finally we consider monolayer graphene sheets rolled into single semiconducting carbon nanotubes (CNT) (Rusin \& Zawadzki, 2007b; Zawadzki, 2006). The band Hamiltonian in the vicinity of $K$ point is (Ajiki \& Ando, 1993)

$$
\hat{H}_{C N T}=u\left(\begin{array}{cc}
0 & \hbar k_{n v}-i \hat{p}_{y} \\
\hbar k_{n v}+i \hat{p}_{y} & 0
\end{array}\right) .
$$

This Hamiltonian is similar to that given by Eq. (16) except that, because of the periodic boundary conditions, the momentum $p_{x}$ is quantized and takes discrete values $\hbar k_{x}=\hbar k_{n v}$, where $k_{n v}=(2 \pi / L)(n-v / 3), n=0, \pm 1, \ldots, v= \pm 1$, and $L$ is the length of circumference of CNT. As a result, the free electron motion can occur only in the direction $y$, parallel to the tube axis. The geometry of CNT has important consequences. There exists an energy gap $E_{g}=2 u \hbar\left|k_{n v}\right|$ and the effective mass at the band edge $m_{0}^{*}=\hbar\left|k_{n v}\right| / u$. For $v= \pm 1$ there always exists a non-vanishing value of the quantized momentum $\hbar k_{n v}$. Finally, for each value of $k_{n v}$ there exists $k_{-n,-v}=-k_{n v}$ resulting in the same subband energy $\mathcal{E}= \pm E$, where

$$
E=\hbar u \sqrt{k_{n v}^{2}+k_{y}^{2}}
$$

The time dependent velocity $\hat{v}_{y}(t)$ and the displacement $\hat{y}(t)$ can be calculated for the plane electron wave in the usual way and they exhibit the ZB oscillations [see Zawadzki (2006)]. For small momenta $k_{y}$ the ZB frequency is $\hbar \omega_{Z}=E_{g}$ and the ZB length is $\lambda_{Z} \approx 1 /\left|k_{n v}\right|$. We are again interested in the displacement $\bar{y}(t)$ of a charge carrier represented by a one-dimensional wave packet analogous to that described in Eq. (3) The average displacement is $\bar{y}(t)=\bar{y}_{Z}(t)-$ $\bar{y}_{\text {sh }}$, where

$$
\bar{y}_{Z}(t)=\frac{\hbar^{2} d u^{2} k_{n v}}{2 \sqrt{\pi}} \int_{-\infty}^{\infty} \frac{d k_{y}}{E^{2}} \cos \left(\frac{2 E t}{\hbar}\right) e^{-d^{2} k_{y}^{2}}
$$

and $\bar{y}_{s h}=1 / 2 \sqrt{\pi} d \operatorname{sgn}(b)[1-\Phi(|b|)] \exp \left(b^{2}\right)$, where $b=k_{n v} d$ and $\Phi(x)$ is the error function. The ZB oscillations of $\bar{y}(t)$ are plotted in Figure 3. It is seen that, after the transient ZB oscillations disappear, there remains a shift $\bar{y}_{s h}$. Thus the ZB separates spatially the charge carriers that are degenerate in energy but characterized by $n, v$ and $-n,-v$ quantum numbers. The current is proportional to $\bar{v}_{y}=\partial \bar{y} / \partial t$, so that the currents related to $v=1$ and $v=-1$ cancel each other. To have a non-vanishing current one needs to break the above symmetry, which can be achieved by applying an external magnetic field parallel to the tube axis.

It is seen from Figure 3 that the decay time of ZB in CNT is much larger than that in bilayer and monolayer graphene. The oscillations decrease proportionally to $t^{-1 / 2}$. The reason is that we consider the situation with $k_{0 y}=0$, so that the ZB oscillations occur due to "built in" momentum $k_{x}=k_{n v}$, arising from the tube's topology. In other words, the long decay time is due to the one-dimensionality of the system. If the circumference of a CNT is increased, the energy gap (and, correspondingly, the ZB frequency) decreases, the amplitude of ZB is larger, but the decay time remains almost unchanged.

One can show that we again deal here with two sub-packets which, however, for $k_{0 y}=0$ do not run away from each other. Thus, the slow damping of $\mathrm{ZB}$ is due only to the slow 


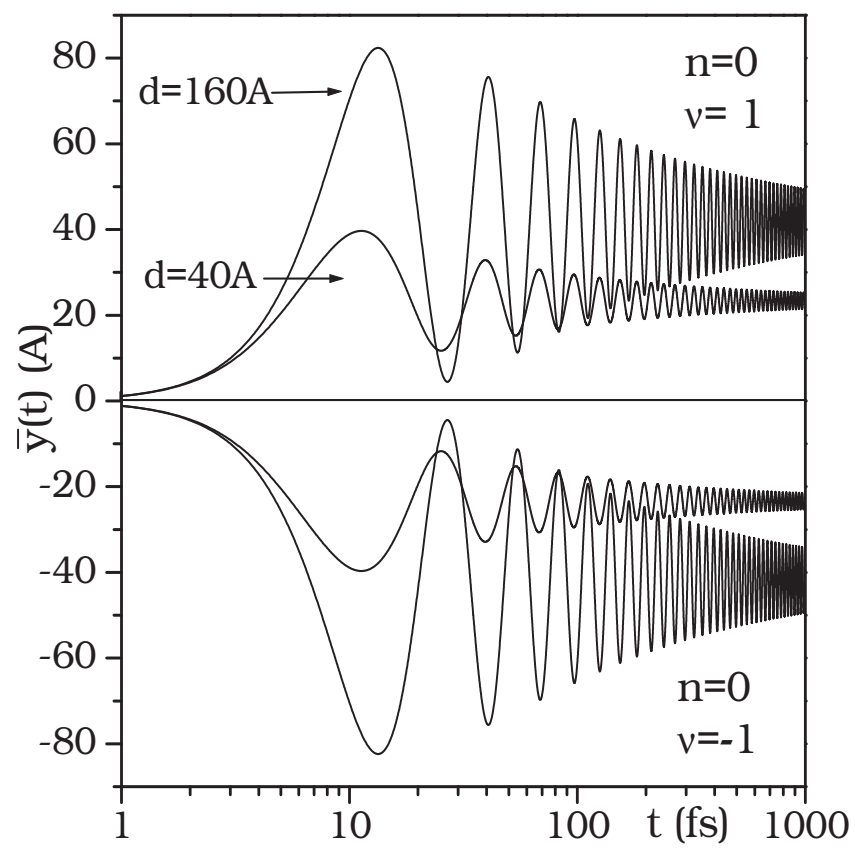

Fig. 3. Zitterbewegung of two charge carriers in the ground subband of a single carbon nanotube of $L=200 \AA$ versus time (logarithmic scale), calculated for gaussian wave packets of two different widths $d$ and $k_{0 y}=0$. After the ZB disappears a constant shift remains. The two carriers are described by different quantum numbers $v$. At higher times the amplitude of ZB oscillations decays as $t^{-1 / 2}$. After Rusin \& Zawadzki (2007b).

broadening of the sub-packets. We emphasize the slow decay, as illustrated in Figure 3, because it is confirmed experimentally, see Gerritsma et al. (2010). We add that for $k_{0 y} \neq 0$ the sub-packets run away from each other and the decay time is much faster.

\section{ZB in graphene in a magnetic field}

The trembling motion of charge carriers in graphene and CNT has been described above for no external potentials. Now we consider the trembling motion of electrons in the presence of an external magnetic field (Rusin \& Zawadzki, 2008). The magnetic field is known to cause no interband electron transitions, so the essential features of $\mathrm{ZB}$ are expected not to be destroyed. On the other hand, introduction of an external field provides an important parameter affecting the $\mathrm{ZB}$ behavior. This case is special because the electron spectrum is fully quantized, so we deal with discrete levels, not bands. We consider a graphene monolayer in an external magnetic field parallel to the $z$ axis. The Hamiltonian for electrons and holes at the $K_{1}$ point of the Brillouin zone is (Slonczewski \& Weiss, 1958; Wallace, 1947)

$$
\hat{H}=u\left(\begin{array}{cc}
0 & \hat{\pi}_{x}-i \hat{\pi}_{y} \\
\hat{\pi}_{x}+i \hat{\pi}_{y} & 0
\end{array}\right)
$$


where $u \approx 1 \times 10^{8} \mathrm{~cm} / \mathrm{s}$ is the characteristic velocity, $\hat{\boldsymbol{\pi}}=\hat{\boldsymbol{p}}-q \hat{\boldsymbol{A}}$ is the generalized momentum, in which $\hat{A}$ is the vector potential and $q$ is the electron charge. Using the Landau gauge, we take $\hat{\boldsymbol{A}}=(-B y, 0,0)$, and for an electron $q=-e$ with $e>0$. We take the wave function in the form $\Psi(x, y)=e^{i k_{x} x} \Phi(y)$. Introducing the magnetic radius $L=\sqrt{\hbar / e B}$, the variable $\xi=y / L-k_{x} L$, and defining the standard raising and lowering operators for the harmonic oscillator $\hat{a}=(\xi+\partial / \partial \xi) / \sqrt{2}$ and $\hat{a}^{\dagger}=(\xi-\partial / \partial \xi) / \sqrt{2}$, the Hamiltonian is rewritten in the form

$$
\hat{H}=-\hbar \Omega\left(\begin{array}{cc}
0 & \hat{a} \\
\hat{a}^{\dagger} & 0
\end{array}\right),
$$

where the frequency is $\Omega=\sqrt{2} u / L$. Next one determines the eigenstates and eigenenergies of the Hamiltonian $\hat{H}$. The energy is $E_{n s}=s \hbar \Omega \sqrt{n}$. Here $n=0,1, \ldots$, and $s= \pm 1$ for the conduction and valence bands, respectively. The above energies were confirmed experimentally. The complete wave function is

$$
|\mathrm{n}\rangle \equiv\left|n k_{x} s\right\rangle=\frac{e^{i k_{x} x}}{\sqrt{4 \pi}}\left(\begin{array}{c}
-s|n-1\rangle \\
|n\rangle
\end{array}\right)
$$

where $|n\rangle$ are the harmonic oscillator functions.

We want to calculate the velocity of a charge carrier described by a wave packet. We first calculate matrix elements $\langle f \mid \mathrm{n}\rangle$ between an arbitrary two-component function $f=\left(f^{u}, f^{l}\right)$ and eigenstates from Eq. (23). A straightforward manipulation gives $\langle f \mid \mathrm{n}\rangle=-s F_{n-1}^{u}+F_{n}^{l}$, where

$$
F_{n}^{j}\left(k_{x}\right)=\frac{1}{\sqrt{2 L} C_{n}} \int g^{j}\left(k_{x}, y\right) e^{-\frac{1}{2} \xi^{2}} \mathrm{H}_{n}(\xi) d y
$$

in which

$$
g^{j}\left(k_{x}, y\right)=\frac{1}{\sqrt{2 \pi}} \int f^{j}(x, y) e^{i k_{x} x} d x
$$

The superscript $j=u, l$ stands for the upper and lower components of the function $f$. The Hamilton equations give the velocity components: $\hat{v}_{i}(0)=\partial \hat{H} / \partial \hat{p}_{i}$, with $i=x, y$. We want to calculate averages of the time-dependent velocity operators $\hat{v}_{i}(t)$ in the Heisenberg picture taken on the function $f$. The averages are

$$
\bar{v}_{i}(t)=\sum_{\mathrm{n}, \mathrm{n}^{\prime}} e^{i E_{\mathrm{n}^{\prime}} t / \hbar}\left\langle f \mid \mathrm{n}^{\prime}\right\rangle\left\langle\mathrm{n}^{\prime}\left|v_{i}(0)\right| \mathrm{n}\right\rangle\langle\mathrm{n} \mid \mathrm{f}\rangle \mathrm{e}^{-\mathrm{i} E_{\mathrm{n}} \mathrm{t} / \hbar},
$$

where the energies and eigenstates are given in Eq. (23). The summation in Eq. (26) goes over all the quantum numbers: $n, n^{\prime}, s, s^{\prime}, k_{x}, k_{x}^{\prime}$. The only non-vanishing matrix elements of the velocity components are for the states states $n^{\prime}=n \pm 1$. One finally obtains

$$
\begin{gathered}
\bar{v}_{y}(t)=u \sum_{n=0}^{\infty} V_{n}^{+} \sin \left(\omega_{n}^{c} t\right)+u \sum_{n=0}^{\infty} V_{n}^{-} \sin \left(\omega_{n}^{Z} t\right)+i u \sum_{n=0}^{\infty} A_{n}^{+} \cos \left(\omega_{n}^{c} t\right)+i u \sum_{n=0}^{\infty} A_{n}^{-} \cos \left(\omega_{n}^{Z} t\right), \\
\bar{v}_{x}(t)=u \sum_{n=0}^{\infty} B_{n}^{+} \cos \left(\omega_{n}^{c} t\right)+u \sum_{n=0}^{\infty} B_{n}^{-} \cos \left(\omega_{n}^{Z} t\right)+i u \sum_{n=0}^{\infty} T_{n}^{+} \sin \left(\omega_{n}^{c} t\right)+i u \sum_{n=0}^{\infty} T_{n}^{-} \sin \left(\omega_{n}^{Z} t\right)
\end{gathered}
$$


where $V_{n}^{ \pm}, T_{n}^{ \pm}, A_{n}^{ \pm}$and $B_{n}^{ \pm}$are given by combinations of $U_{m, n}^{\alpha, \beta}$ integrals

$$
U_{m, n}^{\alpha, \beta}=\int F_{m}^{\alpha *}\left(k_{x}\right) F_{n}^{\beta}\left(k_{x}\right) d k_{x} .
$$

The superscripts $\alpha$ and $\beta$ refer to the upper and lower components, see Rusin \& Zawadzki (2008). In the above model the coefficients $U_{m, n}^{\alpha, \beta}$ are real numbers, so that $A_{n}^{ \pm}$and $T_{n}^{ \pm}$in Eqs. (27) and (28) vanish. The time dependent sine and cosine functions come from the exponential terms in Eq. (26). The frequencies in Eqs. (27) and (28) are $\omega_{n}^{c}=\Omega(\sqrt{n+1}-\sqrt{n})$, $\omega_{n}^{Z}=\Omega(\sqrt{n+1}+\sqrt{n})$, where $\Omega$ is given in Eq. (22). The frequencies $\omega_{n}^{c}$ correspond to the intraband energies while frequencies $\omega_{n}^{Z}$ correspond to the interband energies, see Figure 4 . The interband frequencies are characteristic of the Zitterbewegung. The intraband (cyclotron) energies are due to the band quantization by the magnetic field and they do not appear for non quantized spectra.

Final calculations are carried out for a two-dimensional Gaussian wave packet centered around the wave vector $\mathbf{k}_{\mathbf{0}}=\left(k_{0 x}, 0\right)$ and having two non-vanishing components. In this case one can obtain analytical expressions for $U_{m, n}^{\alpha, \beta}$. The main frequency of oscillations is $\omega_{0}=\Omega$, which can be interpreted either as $\omega_{0}^{c}=\Omega(\sqrt{n+1}-\sqrt{n})$ or $\omega_{0}^{Z}=\Omega(\sqrt{n+1}+\sqrt{n})$ for $n=0$. Frequency $\omega_{0}^{c}$ belongs to the intraband (cyclotron) set, while $\omega_{0}^{Z}$ belongs to the interband set (see Figure 4). The striking feature is, that $\mathrm{ZB}$ is manifested by several frequencies simultaneously. This is a consequence of the fact that in graphene the energy distances between the Landau levels diminish with $n$, which results in different values of frequencies $\omega_{n}^{c}$ and $\omega_{n}^{Z}$ for different $n$. It follows that it is the presence of an external quantizing magnetic field that introduces various frequencies into ZB. It turns out that, after the ZB oscillations seemingly die out, they actually reappear at higher times. Thus, for all $k_{0 x}$ values (including $k_{0 x}=0$ ), the $\mathrm{ZB}$ oscillations have a permanent character, that is they do not disappear in time. This feature is due to the discrete character of the electron spectrum caused by a magnetic field. The above property is in sharp contrast to the no-field cases considered above, in which the spectrum is not quantized and the $\mathrm{ZB}$ of a wave packet has a transient character. In mathematical terms, due to the discrete character of the spectrum, averages of operator quantities taken over a wave packet are sums and not integrals. The sums do not obey the Riemann-Lebesgues theorem for integrals which guarantee the damping of ZB in time for a continuous spectrum see Lock (1979).

Finally, one calculates the displacements $\bar{x}(t)$ and $\bar{y}(t)$ of the wave packet. To this end we integrate Eqs. (27) and (28) with respect to time using the initial conditions $x_{0}=\bar{x}(0)=0$ and $y_{0}=\bar{y}(0)=k_{x} L^{2}$. The results are plotted in Figure 5 in the form of $x-y$ trajectories for different initial wave vectors $k_{0 x}$. The direction of movement is clockwise and the trajectories span early times (1ps) after the creation of a wave packet.

All in all, the presence of a quantizing magnetic field has the following important effects on the trembling motion. (1) For $B \neq 0$ the $\mathrm{ZB}$ oscillation are permanent, while for $B=0$ they are transient. The reason is that for $B \neq 0$ the electron spectrum is discrete. (2) For $B \neq 0$ many $\mathrm{ZB}$ frequencies appear, whereas for $B=0$ only one $\mathrm{ZB}$ frequency exists. (3) For $B \neq 0$ both interband and intraband (cyclotron) frequencies appear in $Z B$; for $B=0$ there are no intraband frequencies. (4) Magnetic field intensity changes not only the $Z B$ frequencies but the entire character of ZB spectrum. 


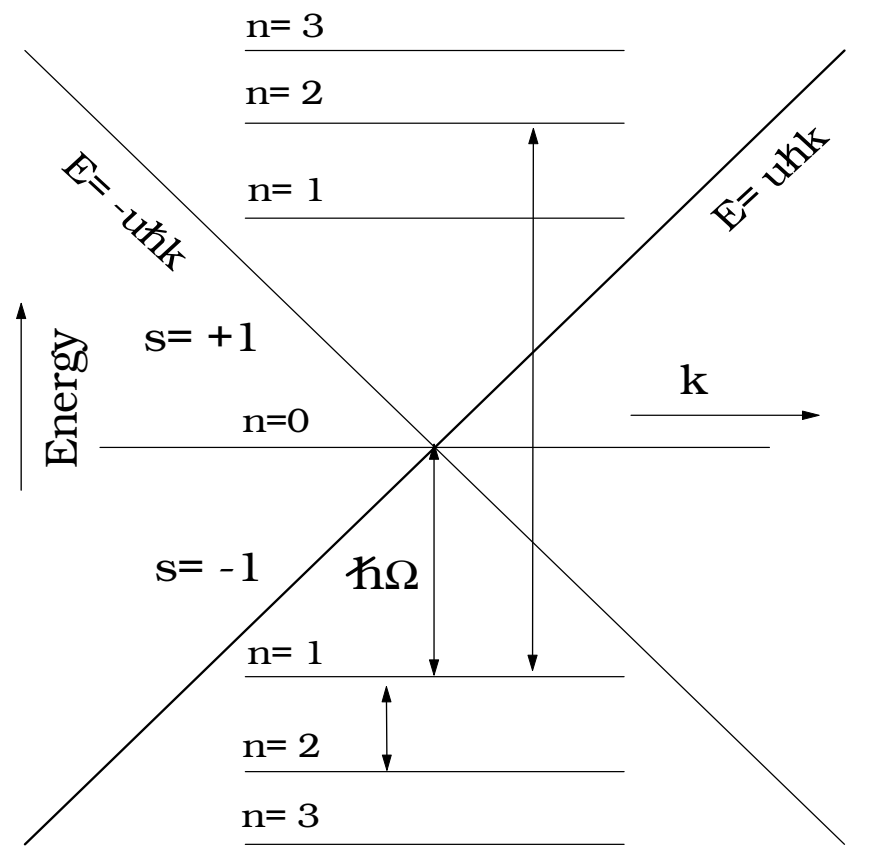

Fig. 4. The energy dispersion $E(k)$ and the Landau levels for monolayer graphene in a magnetic field (schematically). Intraband (cyclotron) and interband (ZB) energies for $n^{\prime}=n \pm 1$ are indicated. The basic energy is $\hbar \Omega=\sqrt{2} \hbar u / L$. After Rusin \& Zawadzki (2008).

The Zitterbewegung should be accompanied by electromagnetic dipole radiation emitted by the trembling electrons. The oscillations $\langle\boldsymbol{r}(t)\rangle$ are related to the dipole moment $-e\langle\boldsymbol{r}(t)\rangle$, which couples to the electromagnetic radiation. One can calculate the emitted electric field from the electron acceleration $\langle\ddot{\boldsymbol{r}}(t\rangle)$ and takes its Fourier transform to determine the emitted frequencies. In Figure 6 we plot the calculated intensities of various emitted lines. The strong peak corresponds to oscillations with the basic frequency $\omega=\Omega$. The peaks on the high-frequency side correspond to the interband excitations and are characteristic of $\mathrm{ZB}$. The peaks on the lower frequency side correspond to the intraband (cyclotron) excitations. In absence of $\mathrm{ZB}$ the emission spectrum would contain only the intraband (cyclotron) frequencies. Thus the interband frequencies $\omega_{n}^{Z}$ shown in Figure 6 are a direct signature of the trembling motion. It can be seen that the $\omega_{z}^{Z}$ peaks are not much weaker than the central peak at $\omega=\Omega$, which means that there exists a reasonable chance to observe them. Generally speaking, the excitation of the system is due to the nonzero momentum $\hbar k_{0 x}$ given to the electron. It can be provided by accelerating the electron in the band or by exciting the electron with a nonzero momentum by light from the valence band to the conduction band. The electron can emit light because the Gaussian wave packet is not an eigenstate of the system described by the Hamiltonian (21). The energy of the emitted light is provided by the initial kinetic energy related to the momentum $\hbar k_{0 x}$. Once this energy is completely used, the emission will cease. 


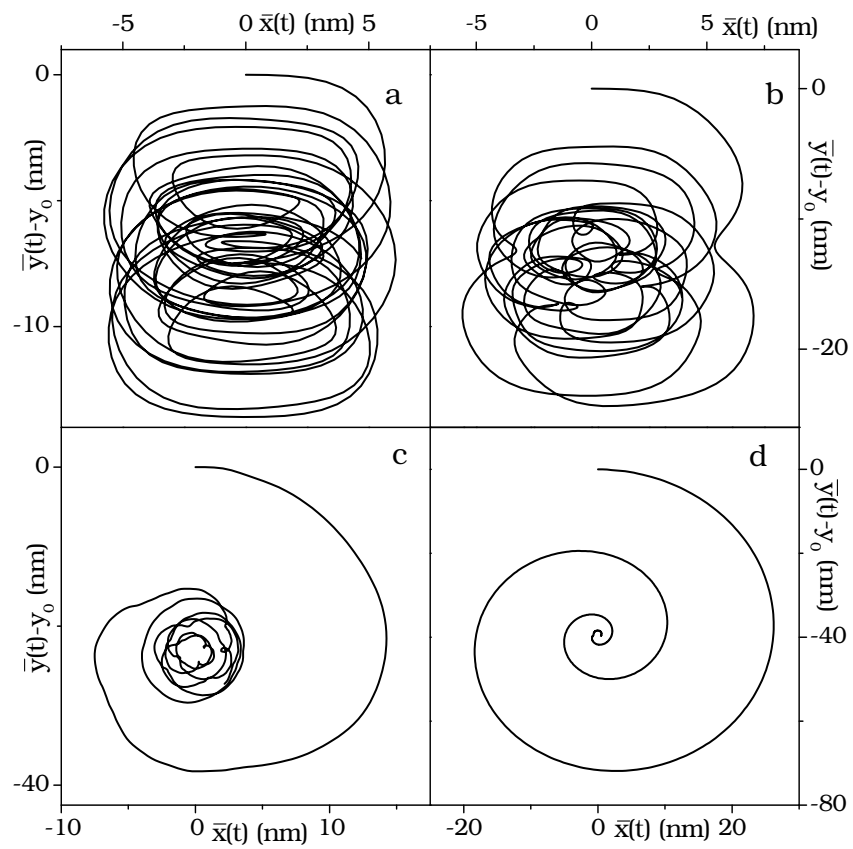

Fig. 5. Zitterbewegung trajectories of electron at the $K_{1}$ point of the Brillouin zone in monolayer graphene at $\mathrm{B}=20 \mathrm{~T}$ during the first picosecond for various values of $k_{0 x}$. After Rusin \& Zawadzki (2008).

Schliemann (2008) described time dependence of the cyclotron motion in monolayer graphene in the presence of a magnetic field using the semiclassical approximation for high carrier energies. He showed that the cyclotron motion is perturbed by interband ZB contributions of higher frequencies. Krueckl \& Kramer (2009) described time propagation of an initially concentrated wave packet in monolayer graphene in a perpendicular magnetic field. A collapse-revival pattern of ZB was investigated and an effect of impurities (disorder) on the packet dynamics was analyzed. It turned out that ZB "survives" the perturbation by impurities. Romera \& de los Santos (2009) studied monolayer graphene in a magnetic field concentrating on collapse-revival pattern of ZB oscillations.

\section{ZB probed by laser pulse}

All the recent theoretical work on $\mathrm{ZB}$ assumed that initially the electrons are represented by Gaussian wave packets. While this assumption represents a real progress compared to the work that had treated electrons as plane waves, it is obviously an idealization since it is not quite clear how to prepare an electron in this form. Here we calculate a reaction of an electron in graphene excited by a laser pulse, not assuming anything about initial form of the electron wave packet. In our description we take into account currently available experimental possibilities.

The following conditions should be met for a successful observation of $\mathrm{ZB}$ : a) The ZB frequency must be in the range of currently detectable regimes, i.e. of the order of $\omega_{Z} \approx 1 \mathrm{fs}{ }^{-1}$, 


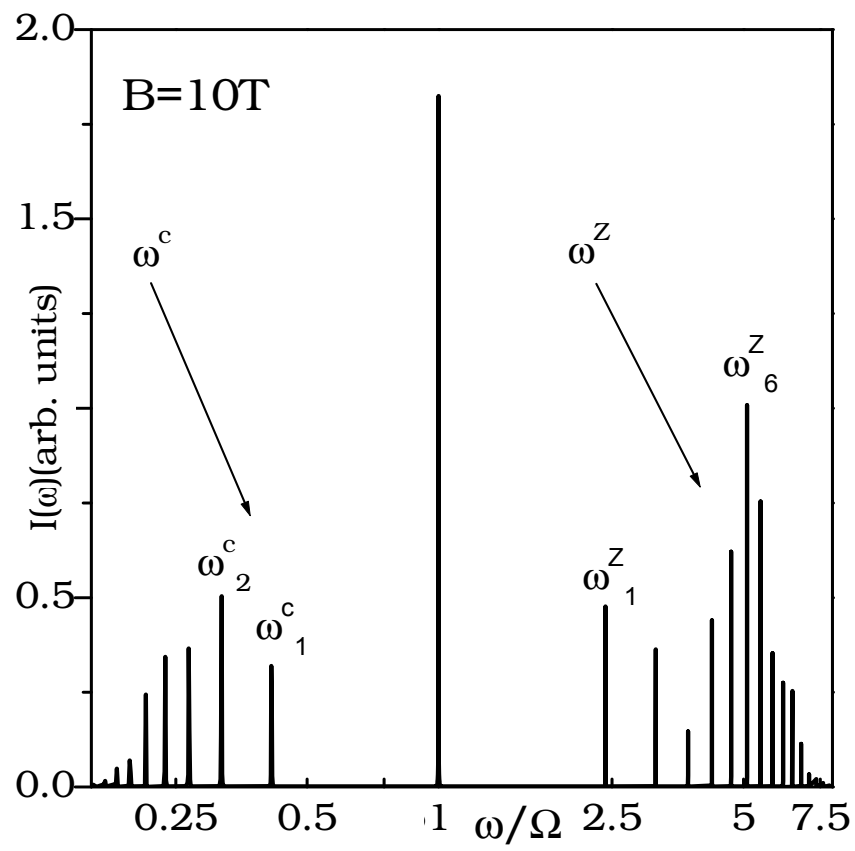

Fig. 6. Intensity spectrum versus frequency during the first $20 \mathrm{ps}$ of motion of an electron described by a Gaussian wave packet having $k_{0 x}=0.035 \AA^{-1}$ in monolayer graphene. After Rusin \& Zawadzki (2008).

and the size of oscillations should be of the order of a few $\AA$; b) The ZB oscillations should be persistent or slowly transient; c) Both positive and negative electron energies must be excited with a sufficient probability; d) The wave packet should be created in a one-electron regime. A system that fulfills the above criteria is p-type monolayer graphene in a constant magnetic field. The wave packet should be created by an ultra short monocycle or sub-monocycle laser pulse. Because of a very wide frequency spectrum of such a pulse, the resulting wave packet will have both positive and negative energies. The electron oscillations give rise to a time-dependent dipole moment which will be a source of electric field and it will emit or absorb radiation in the far infrared range.

In the description we use the above results for the band structure of monolayer graphene in a magnetic field. The Fermi level is assumed to coincide with the Landau level (LL) $n=-2$ and we consider the initial electron to be in the $n=-1$ state. The wavelength of the laser light is assumed to be much larger that the size $L$ of the $n=-1$ state, so we can neglect spatial variation of the electric field in the laser pulse. The total Hamiltonian, including the perturbation due to the laser light, is $\hat{H}_{T}=\hat{H}+\hat{W}(t)$, where $\hat{H}$ is given by Eq. (21) and the perturbing potential due laser light is

$$
\hat{W}(t)=-e y \mathcal{E}_{0} e^{-(2 \ln 2) t^{2} / \tau^{2}} \cos \left(\omega_{L} t\right),
$$

in which $e$ is the electron charge, $\tau$ is the pulse duration (FWHM), $\omega_{L}=2 \pi c / \lambda_{L}$ is the laser frequency (being of the order of $3 \times 10^{15} \mathrm{~s}^{-1}$ ), and $\mathcal{E}_{0}$ is the amplitude of electric field. A 
Gaussian shape of the laser pulse is widely used in optical experiments and it parameterizes effectively a profile of electric field in the laser beam.

As a result of a laser shot, the initial state of the system $\Phi_{k}(t)=\psi_{k} e^{-i E_{k} t / \hbar}$ evolves into the final state $\Psi_{k}(t)=\sum_{j} c_{j}(t) \psi_{k} e^{-i E_{j} t / \hbar}$, which is a combination of the eigenstates of $\hat{H}$ with suitably chosen coefficients $c_{j}(t)$. The resulting time-dependent dipole moment is $\boldsymbol{D}(t)=e\left\langle\Psi_{k}(t)|\boldsymbol{r}| \Psi_{k}(t)\right\rangle$. The corresponding time-dependent wave functions are $\Psi_{k}(t)=$ $e^{-i \hat{H} t / \hbar} \Psi_{k}(0)$, and the dipole moment is

$$
\begin{aligned}
\boldsymbol{D}(t) & =e\left\langle\Psi_{k}(0) e^{i \hat{H} t / \hbar}|\hat{\boldsymbol{r}}| e^{-i \hat{H} t / \hbar} \Psi_{k}(0)\right\rangle \\
& =e\left\langle\Psi_{k}(0)|\hat{\boldsymbol{r}}(t)| \Psi_{k}(0)\right\rangle=e\langle\boldsymbol{r}(t)\rangle .
\end{aligned}
$$

Here $\boldsymbol{r}(t)$ is the electron position in the Heisenberg picture. Thus the dipole moment $\boldsymbol{D}(t)$ is proportional to the time-dependent position averaged over the electron wave packet.

A time-dependent dipole moment is a source of electromagnetic radiation. We treat the radiation classically (Bohm, 1952) and take the radiated transverse electric field to be (Jackson, 1975)

$$
\mathcal{E}_{\perp}(\boldsymbol{r}, t)=\frac{\ddot{\boldsymbol{D}}(t)}{4 \pi \epsilon_{0} c^{2}} \frac{\sin (\theta)}{R},
$$

where $\epsilon_{0}$ is the vacuum permittivity, $\theta$ is an angle between the direction of electron motion and a position of the observer $\boldsymbol{R}$. Since $\ddot{\boldsymbol{D}}(t)=e\langle\ddot{\boldsymbol{r}}(t)\rangle$, Eq. (32) relates the electric field of the dipole with the average acceleration of the packet. If the electric field is measured directly by an antenna, one measures the trembling motion of the wave packet. If the square of electric field is measured in emission or absorption experiments, the signature of $\mathrm{ZB}$ is the existence of peaks corresponding to interband frequencies and their dependence on packet's parameters. Accordingly, in the time resolved luminescence experiments it should be possible to detect directly the motion of the packet with interband frequencies.

The electric field emitted by the trembling electron is calculated by the time-dependent perturbation theory. The induced dipole moment and the corresponding electric field oscillate with three frequencies. The frequency $\omega(|1-\rangle,|2+\rangle)=(\sqrt{2}+1) \Omega$ corresponds to the Zitterbewegung, i.e., to the motion of the packet with an interband frequency. Here $|1-\rangle$ denotes the valence state $n=1$ and $|2+\rangle$ the conduction state $n=2$. The interband frequency is characteristic of $\mathrm{ZB}$ because the trembling motion occurs due to an interference of electron states related to positive and negative electron energies. The second frequency $\omega(|1-\rangle,|2-\rangle)=(\sqrt{2}-1) \Omega$ describes the intraband cyclotron motion of the packet. The third frequency $\omega(|1-\rangle,|0\rangle)=\Omega$ has both interband and intraband character (see Figure 4). In zero-gap materials like graphene the interband $\mathrm{ZB}$ frequency is not much larger than the cyclotron frequency.

In Figure 7 we plot the oscillating dipole moment within the first $1000 \mathrm{fs}$ of motion after the laser shot for two magnetic fields $B=1 \mathrm{~T}$ and $B=10 \mathrm{~T}$, and two laser pulses. In Figure 8 we plot the corresponding electric field for the same parameters during the first 250 fs of oscillations. We assume the laser intensity to be $I=1.0 \times 10^{9} \mathrm{~W} / \mathrm{cm}^{2}$, the emitted electromagnetic wave detected at the angle $\theta=45^{\circ}$, and the distance $R=1 \mathrm{~cm}$. All the quantities in Figures 7 and 8 are calculated per one electron. Since the frequencies are incommensurable, the electron trajectories $\boldsymbol{r}(t)$ are not closed and there is no repeated pattern of oscillations. The motion of the wave packet is permanent in the time scale of femtoseconds 


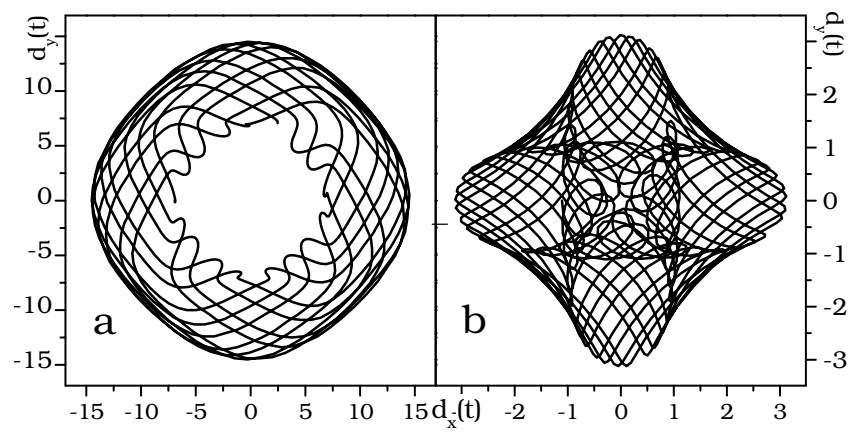

Fig. 7. Oscillations of dipole moment during the first $1000 \mathrm{fs}$ of electron motion after the laser pulse. Experimental characteristics: pulse intensity $1 \times 10^{9} \mathrm{~W} / \mathrm{cm}^{2}$, a) $\tau=1.6 \mathrm{fs}, B=10 \mathrm{~T}, \mathrm{~b}$ ) $\tau=3.0 \mathrm{fs}, B=10 \mathrm{~T}$. Dipole moments in a) are in $10^{-28}[\mathrm{Cm}]$ units, while in $\mathrm{b}$ ) they are in $10^{-31}[\mathrm{Cm}]$ units. The above results refer to very narrow Landau levels, disregarding broadening. After Rusin \& Zawadzki (2009).

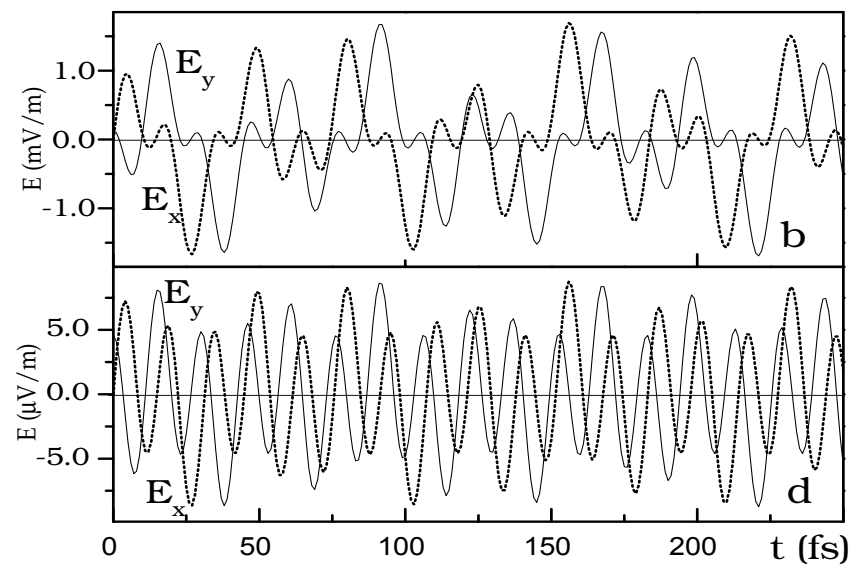

Fig. 8. Electric field emitted by one electron during the first $250 \mathrm{fs}$ of electron motion after the laser pulse. Experimental characteristics: pulse intensity $1 \times 10^{9} \mathrm{~W} / \mathrm{cm}^{2}$, a) $\tau=1.6 \mathrm{fs}, B=10$ $\mathrm{T}, \mathrm{b}) \tau=3.0 \mathrm{fs}, B=10 \mathrm{~T}$. The above results refer to very narrow Landau levels, disregarding broadening. After Rusin \& Zawadzki (2009).

or picoseconds but there is damping of the motion due to the light emission in a long time scale. The results shown in Figures 7 and 8 refer to very narrow Landau levels, disregarding broadening.

We can draw the following qualitative conclusions from Figure 7 and 8. First, for small magnetic fields $B$ the period of oscillations is longer than for large fields, which is related to the basic frequency $\Omega$. Second, irrespective of the variation of $\Omega$ with $B$, for small fields the oscillations are dominated by the low (cyclotron) frequency, while at stronger $B$ the high (ZB) frequency dominates. Finally, comparing the magnitudes of dipole moment or emitted electric field for $\tau=1.6 \mathrm{fs}$ with the corresponding values for $\tau=3.0 \mathrm{fs}$ one observes that the amplitude of oscillations depends very strongly on the duration $\tau$ of the pulse. 


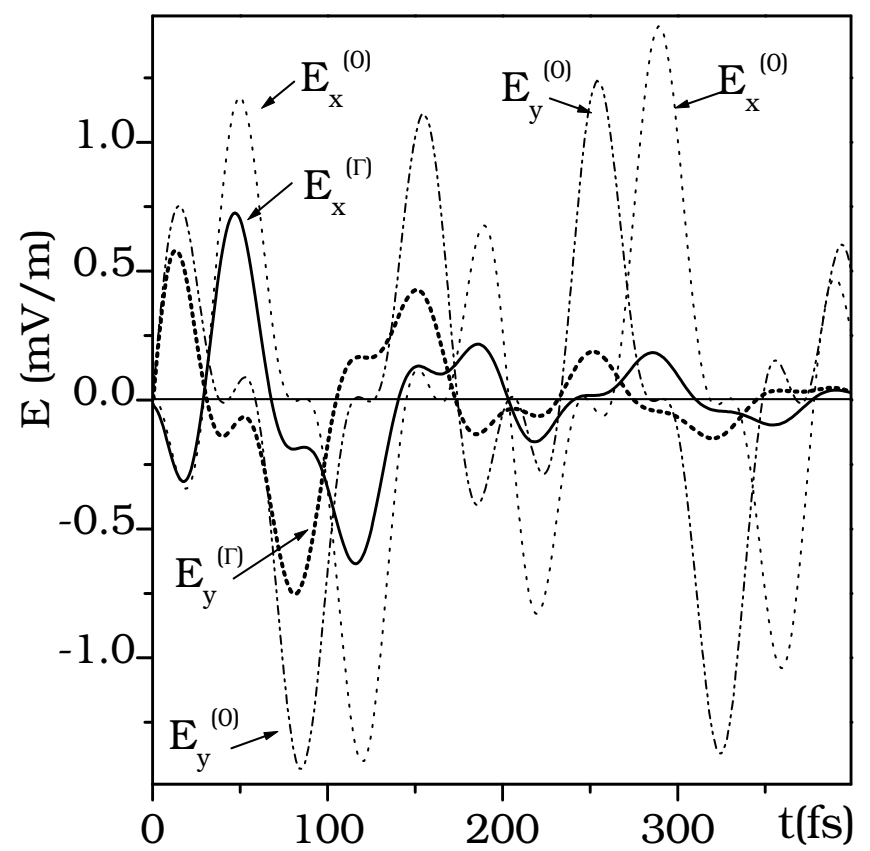

Fig. 9. Calculated electric fields $E_{x}(t)$ and $E_{y}(t)$ emitted by one trembling electron during the first $400 \mathrm{fs}$ after the laser pulse. Pulse parameters: intensity $1 \times 10^{9} \mathrm{~W} / \mathrm{cm}^{2}, \tau=1.6 \mathrm{fs}$, magnetic field $B=1 \mathrm{~T}$. Bold lines - electric fields for broadened Landau levels described by Lorentzian line-shapes with experimental values of $\Gamma_{n}$. Dotted lines - electric fields for delta-like Landau levels. After Rusin \& Zawadzki (2009).

The broadening of the Landau levels may strongly influence the trembling motion of the wave packet. To analyze the overall impact of all the effects leading to the level broadening: disorder, e-e interaction, electron-phonon scattering, etc., one assumes finite widths of all energy levels, characterized by broadening parameters $\Gamma_{n}$. These are treated as phenomenological quantities determined experimentally and including all scattering mechanisms existing in real samples. We approximate the broadening of the density of states by a Lorentzian line-shape irrespective of the detailed scattering mechanism.

The electric field $\boldsymbol{E}(t)$ is calculated, as before, as a second time derivative of $\boldsymbol{D}(t)$. In Figure 9 we plot the electric field emitted by an oscillating electron within the first $400 \mathrm{fs}$ of motion after the laser shot of the width $\tau=1.6 \mathrm{fs}$ in a magnetic field $B=1 \mathrm{~T}$. The two bold lines describe calculated electric fields $E_{x}(t)$ and $E_{y}(t)$ for the Landau levels having the broadening parameters $\Gamma_{n}$ indicated above. The two dotted lines show the electric fields calculated without damping $\left(\Gamma_{n}=0\right)$. Within the first $50 \mathrm{fs}$ of motion the electric fields emitted in the two cases are similar, but later the damping of the emitted fields for broadened levels is visible. After around $400 \mathrm{fs}$ the trembling motion in real case disappears. It can be seen that the maxima of oscillations for the damped ZB motion coincide with the undamped ones. The general conclusion from Figure 9 is that the existence of disorder, many-body effects or other scattering mechanisms changes the persistent $\mathrm{ZB}$ motion to a decaying one, within the characteristic lifetimes for these processes: $\tau_{n}=1 / \Gamma_{n} \approx 130 \mathrm{fs}$. Nevertheless, since the 
parameters $\Gamma_{n}$ used in the calculations correspond to the measured lifetimes in real graphene samples, it follows that the broadening of the Landau levels does not prevent the existence of ZB. Clearly, a lower disorder in better samples will result in longer decay times for ZB.

Wang et al. (2009) carried out a study similar to the one described above, but for bilayer graphene in a magnetic field. This system is somewhat different from monolayer graphene since the Landau levels are nearly uniformly spaced due to quadratic dependence of positive and negative energies on momentum, see Eq. (1). Also, the laser pulse was assumed to contain only one frequency $\omega_{L}$. The authors estimated that in high quality bilayer graphene samples the stimulated ZB electric field can be of the order of volts per meter and the corresponding coherence times of tens of femtoseconds.

\section{Discussion and conclusions}

In spite of the great interest in the phenomenon of $Z B$ its physical origin remained mysterious. As mentioned above, it was recognized that the $\mathrm{ZB}$ in a vacuum is due to an interference of states corresponding to positive and negative electron energies. Since the ZB in solids was treated by two-band Hamiltonians similar to the Dirac equation, its interpretation was also similar. This did not explain its origin, it only provided a way to describe it. For this reason we considered recently the fundamentals of electron propagation in a periodic potential (Zawadzki \& Rusin, 2010). The physical origin of ZB is essential because it resolves the question of its observability. The second purpose was to decide whether the two-band k.p model of the band structure, used to describe the ZB in solids, was adequate.

It is often stated that an electron moving in a periodic potential behaves like a free particle characterized by an effective mass $m^{*}$. The above picture suggests that, if there are no external forces, the electron moves in a crystal with a constant velocity. This, however, is clearly untrue because the electron velocity operator $\hat{v}_{i}=\hat{p}_{i} / m_{0}$ does not commute with the Hamiltonian $\hat{H}=\hat{\mathbf{p}}^{2} / 2 m_{0}+V(\mathbf{r})$, so that $\hat{v}_{i}$ is not a constant of the motion. In reality, as the electron moves in a periodic potential, it accelerates or slows down keeping its total energy constant. This situation is analogous to that of a roller-coaster: as it goes down losing its potential energy, its velocity (i.e. its kinetic energy) increases, and when it goes up its velocity decreases. We first considered the trembling frequency $\omega_{Z}$. The latter is easy to determine if we assume, in the first approximation, that the electron moves with a constant average velocity $\bar{v}$ and the period of the potential is $a$, so $\omega_{Z}=2 \pi \bar{v} / a$. Putting typical values for GaAs: $a=5.66 \AA, \bar{v}=2.3 \times 10^{7} \mathrm{~cm} / \mathrm{s}$, one obtains $\hbar \omega_{Z}=1.68 \mathrm{eV}$, i.e. the interband frequency since the energy gap is $E_{g} \simeq 1.5 \mathrm{eV}$. The interband frequency is in fact typical for the $\mathrm{ZB}$ in solids. One can go further calculating classical velocity oscillations for a periodic potential using a condition of the energy conservation: $m v_{z}^{2} / 2+e V(z)=E$, which for a periodic potential $V(z)$ gives velocity oscillations. Finally, we used a quantum approach employing the Kronig-Penney delta-like potential since it allows one to calculate explicitly the eigenenergies and eigenfunctions (Kronig \& Penney, 1931; Smith, 1961). The oscillations of the packet velocity calculated directly from the periodic potential have many similarities to those computed on the basis of the two-band k.p model. The question arises: does one deal with the same phenomenon in the two cases? To answer this question we calculated ZB using the two methods for the same periodic potential. It turned out that the two-band k.p model gives an excellent description of $\mathrm{ZB}$ for instantaneous velocities. This agreement demonstrates that the theories based on: (a) the periodic potential and (b) the band structure, describe the 
same trembling motion of the electron. The procedure based on the energy band structure is more universal since it also includes cases which do not seem to have a direct classical interpretation. The main conclusion is that the electron Zitterbewegung in crystalline solids is the basic way of electron propagation in a periodic potential. The established nature of $\mathrm{ZB}$ indicates that the latter should certainly be observable. Another important conclusion is that, in spite of similarities between electron ZB in a vacuum and in crystalline solids, the origin of the phenomenon in the two situations is completely different.

Recently there appeared many propositions to simulate the Dirac equation and the resulting phenomena with the use of other systems. Since in narrow-gap semiconductors and in graphene one deals with the Hamiltonians resembling the Dirac equation, one can apply simulations also to electrons in these materials. Many simulations make use of trapped atoms or ions interacting with laser light. There are two essential advantages of such methods. First, it is possible to follow the interaction of laser light with few or even single atoms or ions. Second, when simulating the Hamiltomians it is possible to modify their basic parameters in order to make the ZB frequency much lower and its amplitude much larger. In consequence, they become measurable with current experimental techniques. Simulations of important Hamiltonians are carried out with the use of Jaynes-Cummings model (Johanning et al., 2009; Lamata et al., 2007; Leibfried et al., 2003) known from quantum and atomic optics. In particular, Gerritsma et al. (2010) simulated for the first time the 1+1 Dirac equation with the resulting one-dimensional Zitterbewegung using ${ }^{40} \mathrm{Ca}^{+}$trapped ions. The results agree very well with the predictions of Zawadzki \& Rusin (2010). The reason of this agreement is that the theory of Zawadzki \& Rusin (2010), while concerned with semiconductors, also uses an effective Dirac equation. Gerritsma et al. (2010) showed that, if the wave packet does not have the initial momentum, the decay time of ZB is much slower. This agrees with theoretical results for carbon nanotubes, as shown in our Figure 3.

An important recognition won after the considerable effort of the last years is, that the Zitterbewegung is not a marginal, obscure and probably unobservable effect, but a real and universal phenomenon that often occurs in both quantum and non-quantum systems. Clearly, the ZB in a vacuum proposed by Schroedinger (1930) stands out as an exception since it is supposed to occur without any external force. However, in its original form it is probably not directly observable for years to come and one has to recourse to its simulations. On the other hand, manifestations of ZB in crystalline solids and other periodic systems turned out to be quite common and they are certainly observable. A universal background underlying the phenomenon of $\mathrm{ZB}$ in any system (including a vacuum) is an interference of states belonging to positive and negative energies (in a generalized sense, see below). The positive and negative energies belong usually to bands but they can also be discrete levels, as shown for electrons in graphene in a magnetic field, see Figure 4.

A question arises: what should be called "Zitterbewegung"? It seems that the signature of ZB phenomenon is its interband frequency, in which the term interband has the meaning "between interacting bands". An instructive example is provided by graphene in a magnetic field (see Figure 4), where the electron motion contains both intraband and interband frequencies. We believe that only the interband contributions should be called $\mathrm{ZB}$, while the intraband ones are simply the cyclotron components. It appears that the second signature of $\mathrm{ZB}$ is the actual motion which, for instance, distinguishes it from the Rabi oscillations.

If an electron is prepared in the form of a wave packet, and if the electron spectrum is not completely quantized, the ZB has a transient character, i.e. it decays in time. One can 
show that the decay time is inversely proportional to the momentum spread $\Delta k$ of the wave packet, see Eq. (6). Physically, the transient character of ZB comes about as a result of waning interference of the two sub-packets belonging to positive and negative energies as they go apart because of different speeds, see Eqs. (14) and (15). The decay time is usually much longer in one-dimensional systems, see Figure 3 and Rusin \& Zawadzki (2007b). On the other hand, if the electron spectrum is discrete, ZB persists in time, sometimes in the form of collapse-revival patterns. In general, the wave packet should have a non-vanishing initial momentum in one direction to exhibit the $\mathrm{ZB}$ in the perpendicular direction, but this is not always the case.

One should ask the question about possible observation of Zitterbewegung in graphene. Two different ways were proposed to observe the trembling electrons. The first is to detect an ac current related to the $\mathrm{ZB}$ velocity, see Figure 2. One needs a current meter sensitive to the $\mathrm{ZB}$ frequency. Then, even if the electrons do not move in phase so that the net current averages to zero, the meter should detect a clear increase of noise at the frequency $\omega_{Z}$. The second possible way to observe the $\mathrm{ZB}$ is to detect electromagnetic radiation emitted by the trembling electrons, see Figure 6 and Rusin \& Zawadzki (2008). The emission is possible because, if the electrons are prepared in form of wave packets or they respond to light wave packets, they are not in their eigenstates. Castro Neto et al. (2009) describing electronic properties of graphene considered possible manifestations of $\mathrm{ZB}$ in electron transport due to confinement of electron motion. The proposed ZB should not be confused with the Bloch oscillations of charge carriers in superlattices. The Bloch oscillations are basically a one-band phenomenon and they require an external electric field driving electrons all the way to the Brillouin zone boundary. On the other hand, the ZB needs at least two bands and it is a no-field phenomenon. Narrow gap superlattices and graphene can provide a suitable system for its observation.

\section{Appendix A}

In this appendix we describe the time evolution of electrons in monolayer graphene in the presence of a magnetic field using operator techniques rather than averages. The initial Hamiltonian for the problem reads

$$
\hat{H}=u \sigma_{x} \hat{\pi}_{x}+u \sigma_{y} \hat{\pi}_{y}
$$

where $\hat{\pi}=\hat{p}-q \boldsymbol{A}$ is the generalized momentum, $q$ is the electron charge, $\sigma_{i}$ are Pauli matrices. Taking the magnetic field $\boldsymbol{B} \| \boldsymbol{z}$ we choose the vector potential $\boldsymbol{A}=(-B y, 0)$. For an electron there is $q=-e$ with $e>0$. One can look for solutions of the Schrodinger equation in the form

$$
\Psi(\boldsymbol{r})=e^{i k_{x} x} \Phi(y),
$$

which gives an effective Hamiltonian $\hat{H}$

$$
\hat{H}=c \hbar\left[\left(k_{x}-e B y / \hbar\right) \sigma_{x}+(\partial / i \partial y) \sigma_{y}\right] .
$$

Introducing the magnetic radius $L=\sqrt{\hbar / e B}$ and $\xi=y / L-k_{x} L$ one has $y=\xi L+k_{x} L^{2}$, $e B / \hbar=1 / L^{2}$, and $\partial / \partial y=(1 / L) \partial / \partial \xi$. Defining the standard raising and lowering operators for the harmonic oscillator

$$
\left\{\begin{array}{c}
\hat{a}=(\xi+\partial / \partial \xi) / \sqrt{2} \\
\hat{a}^{+}=(\xi-\partial / \partial \xi) / \sqrt{2}
\end{array}\right.
$$


one has $\left[\hat{a}, \hat{a}^{+}\right]=1$ and $\xi=\left(\hat{a}+\hat{a}^{+}\right) / \sqrt{2}$. The rewritten Hamiltonian $\hat{H}$ is

$$
\hat{H}=-\hbar \omega\left(\begin{array}{cc}
0 & \hat{a} \\
\hat{a}^{+} & 0
\end{array}\right)
$$

with $\omega=\sqrt{2} c / L$. The frequency $\omega$ is often used in our considerations.

Now we introduce an important two-component operator $\hat{A}=\operatorname{diag}(\hat{a}, \hat{a})$ and its adjoint operator $\hat{A}^{+}=\operatorname{diag}\left(\hat{a}^{+}, \hat{a}^{+}\right)$. Next we define the two-component position operators

$$
\begin{aligned}
& \hat{Y}=\frac{L}{\sqrt{2}}\left(\hat{A}+\hat{A}^{+}\right), \\
& \hat{X}=\frac{L}{i \sqrt{2}}\left(\hat{A}-\hat{A}^{+}\right),
\end{aligned}
$$

in analogy to the position operators $\hat{y}$ and $\hat{x}$. We intend to calculate the time dependence of $\hat{A}$ and $\hat{A}^{+}$and then the time dependence of $\hat{Y}$ and $\hat{X}$.

To find the dynamics of $\hat{A}$ one calculates the first and second time derivatives of $\hat{A}$ using the equation of motion: $\hat{A}_{t} \equiv d \hat{A} / d t=(i / \hbar)[\hat{H}, \hat{A}]$. We have

$$
\begin{aligned}
& \hat{A}_{t}=i \omega\left(\begin{array}{ll}
0 & 0 \\
1 & 0
\end{array}\right) \\
& \hat{A}_{t}^{+}=-i \omega\left(\begin{array}{ll}
0 & 1 \\
0 & 0
\end{array}\right) .
\end{aligned}
$$

The second time derivatives of $\hat{A}$ and $\hat{A}^{+}$are calculated following the trick proposed by Schrodinger. We use two versions of this trick

$$
\begin{aligned}
& \hat{A}_{t t}=(i / \hbar)\left[\hat{H}, \hat{A}_{t}\right]=\frac{2 i}{\hbar} \hat{H} \hat{A}_{t}-\frac{i}{\hbar}\left\{\hat{H}, \hat{A}_{t}\right\}, \\
& \hat{A}_{t t}^{+}=(i / \hbar)\left[\hat{H}, \hat{A}_{t}^{+}\right]=-\frac{2 i}{\hbar} \hat{A}_{t}^{+} \hat{H}+\frac{i}{\hbar}\left\{\hat{H}, \hat{A}_{t}^{+}\right\} .
\end{aligned}
$$

There is $(i / \hbar)\left\{\hat{H}, \hat{A}_{t}\right\}=\omega^{2} \hat{A}$ and $(i / \hbar)\left\{\hat{H}, \hat{A}_{t}^{+}\right\}=-\omega^{2} \hat{A}^{+}$. In consequence we obtain second order equations for $\hat{A}$ and $\hat{A}^{+}$

$$
\begin{aligned}
& \hat{A}_{t t}=(2 i / \hbar) \hat{H} \hat{A}_{t}-\omega^{2} \hat{A} \\
& \hat{A}_{t t}^{+}=-(2 i / \hbar) \hat{A}_{t}^{+} \hat{H}-\hat{A}^{+} \omega^{2} .
\end{aligned}
$$

To solve the above equations one eliminates the terms with the first derivative using the substitutions $\hat{A}=\exp (+i \hat{H} t / \hbar) \hat{B}$ and $\hat{A}^{+}=\hat{B}^{+} \exp (-i \hat{H} t / \hbar)$, which gives

$$
\begin{aligned}
& \hat{B}_{t t}=-\left(1 / \hbar^{2}\right) \hat{H}^{2} \hat{B}-\omega^{2} \hat{B}, . \\
& \hat{B}_{t t}^{+}=-\left(1 / \hbar^{2}\right) \hat{B}^{+} \hat{H}^{2}-\hat{B}^{+} \omega^{2} .
\end{aligned}
$$


Finally

$$
\begin{aligned}
& \hat{B}_{t t}=-\left(\hat{\Omega}^{2}+\omega^{2}\right) \hat{B}, \\
& \hat{B}_{t t}^{+}=-\hat{B}^{+}\left(\hat{\Omega}^{2}+\omega^{2}\right),
\end{aligned}
$$

where $\hat{\Omega}=\hat{H} / \hbar$. The solutions of the above equations are

$$
\begin{gathered}
\hat{B}=e^{-i \hat{M} t} \hat{C}_{1}+e^{i \hat{M} t} \hat{C}_{2}, \\
\hat{B}^{+}=\hat{C}_{1}^{+} e^{-i \hat{M} t}+\hat{C}_{2}^{+} e^{i \hat{M} t},
\end{gathered}
$$

where $\hat{M}=+\sqrt{\hat{\Omega}^{2}+\omega^{2}}$ is the positive root of $\hat{M}^{2}=\hat{\Omega}^{2}+\omega^{2}$. The operator $\hat{M}$ is an important quantity in our considerations. Both $\hat{C}_{1}$ and $\hat{C}_{2}^{+}$are time-independent operators. Coming back to $\hat{A}(t)$ and $\hat{A}^{+}(t)$ one has

$$
\begin{gathered}
\hat{A}(t)=e^{i \hat{\Omega} t} e^{-i \hat{M} t} \hat{C}_{1}+e^{i \hat{\Omega} t} e^{+i \hat{M} t} C_{2} \\
\hat{A}^{+}(t)=\hat{C}_{1}^{+} e^{+i \hat{M} t} e^{-i \hat{\Omega} t}+\hat{C}_{2}^{+} e^{-i \hat{M} t} e^{-i \hat{\Omega} t} .
\end{gathered}
$$

In order to find the final forms of $\hat{A}(t)$ and $\hat{A}^{+}(t)$ one has to use the initial conditions. They are

$$
\begin{aligned}
\hat{A}(0) & =\hat{C}_{1}+\hat{C}_{2}, \\
\hat{A}^{+}(0) & =\hat{C}_{1}^{+}+\hat{C}_{2}^{+}, \\
\hat{A}_{t}(0) & =i(\hat{\Omega}-\hat{M}) \hat{C}_{1}+i(\hat{\Omega}+\hat{M}) \hat{C}_{2} \\
\hat{A}_{t}^{+}(0) & =-i \hat{C}_{1}^{+}(\hat{\Omega}-\hat{M})-i \hat{C}_{2}^{+}(\hat{\Omega}+\hat{M}) .
\end{aligned}
$$

Simple manipulations give

$$
\begin{aligned}
& \hat{C}_{1}=\frac{i}{2} \hat{M}^{-1} \hat{A}_{t}(0)+\frac{1}{2} \hat{M}^{-1} \hat{\Omega} \hat{A}(0)+\frac{1}{2} \hat{A}(0), \\
& \hat{C}_{2}=-\frac{i}{2} \hat{M}^{-1} \hat{A}_{t}(0)-\frac{1}{2} \hat{M}^{-1} \hat{\Omega} \hat{A}(0)+\frac{1}{2} \hat{A}(0) .
\end{aligned}
$$

Similarly

$$
\begin{aligned}
& \hat{C}_{1}^{+}=-\frac{i}{2} \hat{A}_{t}^{+}(0) \hat{M}^{-1}+\frac{1}{2} \hat{A}^{+}(0) \hat{\Omega} \hat{M}^{-1}+\frac{1}{2} \hat{A}^{+}(0), \\
& \hat{C}_{2}^{+}=\frac{i}{2} \hat{A}_{t}^{+}(0) \hat{M}^{-1}-\frac{1}{2} \hat{A}^{+}(0) \hat{\Omega} \hat{M}^{-1}+\frac{1}{2} \hat{A}^{+}(0) .
\end{aligned}
$$

One can see by inspection that the initial conditions for $\hat{A}(0)$ and $\hat{A}_{t}(0)$ are satisfied. It is convenient to express $\hat{A}_{t}$ in terms of $\hat{A}$ and $\hat{\Omega}$ using the equation of motion $i \hat{A}_{t}=\hat{A} \hat{\Omega}-\hat{\Omega} \hat{A}$. Then the first and second terms in Eqs. (54) and (56) partially cancel out and the operator $\hat{A}(t)$ 
can be expressed as a sum $\hat{A}(t)=\hat{A}_{1}(t)+\hat{A}_{2}(t)$, where

$$
\begin{aligned}
& \hat{A}_{1}(t)=\frac{1}{2} e^{i \hat{\Omega} t} e^{-i \hat{M} t}\left[\hat{A}(0)+\hat{M}^{-1} \hat{A}(0) \hat{\Omega}\right], \\
& \hat{A}_{2}(t)=\frac{1}{2} e^{i \hat{\Omega} t} e^{+i \hat{M} t}\left[\hat{A}(0)-\hat{M}^{-1} \hat{A}(0) \hat{\Omega}\right] .
\end{aligned}
$$

Similarly, one can break $\hat{A}^{+}(t)=\hat{A}_{1}^{+}(t)+\hat{A}_{2}^{+}(t)$, where

$$
\begin{aligned}
& \hat{A}_{1}^{+}(t)=\frac{1}{2}\left[\hat{A}^{+}(0)+\hat{\Omega} \hat{A}^{+}(0) M^{-1}\right] e^{+i \hat{M} t} e^{-i \hat{\Omega} t}, \\
& \hat{A}_{2}^{+}(t)=\frac{1}{2}\left[\hat{A}^{+}(0)-\hat{\Omega} \hat{A}^{+}(0) M^{-1}\right] e^{-i \hat{M} t} e^{-i \hat{\Omega} t} .
\end{aligned}
$$

Using Eqs. (38) and (39) we obtain

$$
\begin{aligned}
& \hat{Y}(t)=\frac{L}{\sqrt{2}}\left(\hat{A}_{1}(t)+\hat{A}_{2}(t)+\hat{A}_{1}^{+}(t)+\hat{A}_{2}^{+}(t)\right), \\
& \hat{X}(t)=\frac{L}{i \sqrt{2}}\left(\hat{A}_{1}(t)+\hat{A}_{2}(t)-\hat{A}_{1}^{+}(t)-\hat{A}_{2}^{+}(t)\right) .
\end{aligned}
$$

The above compact equations are final expressions for the time dependence of $\hat{A}(t)$ and $\hat{A}^{+}(t)$ operators and, by means of Eqs. (62) and (63), for the time dependence of the position operators $\hat{Y}(t)$ and $\hat{X}(t)$. These exact results are given in terms of operators $\hat{\Omega}$ and $\hat{M}$. To finalize this description one needs to specify the physical sense of functions of these operators appearing in Eqs. (58)-(63).

As it will be seen below, operators $\hat{\Omega}$ and $\hat{M}$ have the same eigenfunctions, so they commute. Then the product of two exponential functions in Eqs. (58)-(61) is given by the exponential function with the sum of two exponents. In consequence, there appear two sets of frequencies $\omega^{+}$and $\omega^{-}$corresponding to the sum and the difference: $\omega^{-} \sim \hat{M}-\hat{\Omega}$, and $\omega^{+} \sim \hat{M}+\hat{\Omega}$, respectively. The first frequencies $\omega^{-}$, being of the intraband type, lead in the non-relativistic limit to the cyclotron frequency $\omega_{c}$. The interband frequencies $\omega^{+}$ correspond to the Zitterbewegung. The electron motion is a sum of different frequency components. In absence of a magnetic field there are no intraband frequencies and only one interband frequency. Each of the operators $\hat{A}(t)$ or $\hat{A}^{+}(t)$ contains both intraband and interband terms. One could infer from Eqs. (59) and (61) that the amplitudes of interband and intraband terms are similar. However, when the explicit forms of the matrix elements of $\hat{A}(t)$ and $\hat{A}^{+}(t)$ are calculated, it turns out that the ZB terms are much smaller than the cyclotron terms, except at high magnetic fields. The operators $\hat{\Omega}$ and $\hat{M}$ do not commute with $\hat{A}$ or $\hat{A}^{+}$. In Eq. (59) the operator $\hat{A}$ acts on the exponential terms from the right-hand side, while in Eq. (61) the operator $\hat{A}^{+}$acts from the left-hand side.

Let us consider the operator $\hat{M}^{2}=\hat{\Omega}^{2}+\omega^{2}$. Let $E_{\mathrm{n}} / \hbar$ and $|\mathrm{n}\rangle$ be the eigenvalue and eigenvector of $\hat{\Omega}$, respectively. Then

$$
\hat{M}^{2}|\mathrm{n}\rangle=\left(\hat{\Omega}^{2}+\omega^{2}\right)|\mathrm{n}\rangle=\frac{1}{\hbar^{2}}\left(E_{\mathrm{n}}^{2}+\hbar^{2} \omega^{2}\right)|\mathrm{n}\rangle .
$$


Thus, every state $|\mathrm{n}\rangle$ is also an eigenstate of the operator $\hat{M}^{2}$ with the eigenvalue $\lambda_{\mathrm{n}}^{2}=$ $E_{\mathrm{n}}^{2} / \hbar^{2}+\omega^{2}$. To find a more convenient form of $\lambda_{\mathrm{n}}$ we must find an explicit form of $E_{\mathrm{n}}$. To do this we choose again the Landau gauge $\boldsymbol{A}=(-B y, 0)$. Then, the eigenstate $|\mathrm{n}\rangle$ is characterized by three quantum numbers: $n, k_{x}, s$, where $n$ is the harmonic oscillator number, $k_{x}$ is the wave vector in $x$ direction and $s= \pm 1$ labels the positive and negative energy branches. The state $|\mathrm{n}\rangle$ in a magnetic field is

$$
|\mathrm{n}\rangle=\frac{e^{i k_{x} x}}{N_{n}}\left(\begin{array}{c}
-s|n-1\rangle \\
|n\rangle
\end{array}\right),
$$

The energies of electrons in graphene are $E_{n s}=s \hbar \omega \sqrt{n}$ and the norm is $N_{n}=4 \pi$ for $n>0$ and $N_{0}=2 \pi$. The harmonic oscillator states are

$$
\langle\boldsymbol{r} \mid n\rangle=\frac{1}{2 \pi \sqrt{L} C_{n}} \mathrm{H}_{n}(\xi) e^{-1 / 2 \xi^{2}},
$$

where $\mathrm{H}_{n}(\xi)$ are the Hermite polynomials and $C_{n}=\sqrt{2^{n} n ! \sqrt{\pi}}$. Using the above forms for $|\mathrm{n}\rangle$ and $E_{n s}$ we obtain from Eq. (64)

$$
\hat{M}^{2}|\mathrm{n}\rangle=\omega(n+1)|\mathrm{n}\rangle,
$$

i.e. $\lambda_{n}= \pm \omega \sqrt{n+1}= \pm \omega_{n+1}$, where $\omega_{n}=\omega \sqrt{n}$. In further calculations we assume $\lambda_{n}$ to be positive. The operator $\hat{M}^{2}$ is diagonal and its explicit form is $\hat{M}^{2}=\operatorname{diag}\left[\hat{a} \hat{a}^{+}, \hat{a}^{+} \hat{a}\right]$, see Eq. (64). Because $\hat{M}^{2}=\Omega^{2}+\omega^{2}$, the eigenstates of $\hat{M}^{2}$ do not depend on the energy branch index $s$.

To calculate the functions of operators $\hat{\Omega}$ and $\hat{M}$ we use the fact that, for every reasonable function $f$ of operators $\hat{\Omega}$ or $\hat{M}^{2}$ there is $f(\hat{\Omega})=\sum_{\mathrm{n}} f\left(s \omega_{n}\right)|\mathrm{n}\rangle\langle\mathrm{n}|$, and $f\left(\hat{M}^{2}\right)=$ $\sum_{\mathrm{n}} f\left(\lambda_{n}^{2}\right)|\mathrm{n}\rangle\langle\mathrm{n}|$, see e.g Feynmann (1972). Thus

$$
\begin{aligned}
e^{ \pm i \hat{\Omega} t} & =\sum_{\mathrm{n}} e^{ \pm i s t \omega_{n}}|\mathrm{n}\rangle\langle\mathrm{n}|, \\
\hat{M} & =\left(\hat{M}^{2}\right)^{1 / 2}=v \sum_{\mathrm{n}} \lambda_{n}|\mathrm{n}\rangle\langle\mathrm{n}|, \\
\hat{M}^{-1} & =\left(\hat{M}^{2}\right)^{-1 / 2}=v \sum_{\mathrm{n}} \frac{1}{\lambda_{n}}|\mathrm{n}\rangle\langle\mathrm{n}|, \\
e^{ \pm i \hat{M} t} & =e^{ \pm i t\left(\hat{M}^{2}\right)^{1 / 2}}=\sum_{\mathrm{n}} e^{ \pm i v t \lambda_{n}}|\mathrm{n}\rangle\langle\mathrm{n}|,
\end{aligned}
$$

where $v= \pm 1$. Without loss of generality we take $v=+1$. The above formulas can be used in calculating the matrix elements of $\hat{A}(t)$ and $\hat{A}^{+}(t)$.

Taking the eigenvectors $|\mathrm{n}\rangle=\left|n, k_{x}, s\right\rangle$ and $\left|\mathrm{n}^{\prime}\right\rangle=\left|n^{\prime}, k_{x}^{\prime}, s^{\prime}\right\rangle$ with $n^{\prime}=n+1$, we calculate matrix elements $\hat{A}_{\mathrm{n}, \mathrm{n}^{\prime}}(t)$ using $\hat{A}(t)$ given in Eqs. (58) and (59). The selection rules for $\hat{A}_{\mathrm{n}, \mathrm{n}^{\prime}}(0)$ are $k_{x}=k_{x}^{\prime}$, while there are no selection rules for $s, s^{\prime}$. The matrix element of $\hat{M}^{-1} \hat{A}(0) \hat{\Omega}$ appearing in Eqs. (58) and (59) is

$$
\left\langle\mathrm{n}\left|\hat{M}^{-1} \hat{A}(0) \hat{\Omega}\right| \mathrm{n}^{\prime}\right\rangle=\frac{1}{\lambda_{n}} \hat{A}(0)_{\mathrm{n}, \mathrm{n}^{\prime}} s^{\prime} \omega_{n^{\prime}}=s^{\prime} \hat{A}(0)_{\mathrm{n}, \mathrm{n}^{\prime}} .
$$


In the last equality we used $\omega_{n^{\prime}}=\omega_{n+1}$ and $\lambda_{n}=\omega \sqrt{n+1}$. Then

$$
\begin{aligned}
& \hat{A}_{1}(t)_{\mathrm{n}, \mathrm{n}^{\prime}}=\frac{1}{2} e^{i\left(s \omega_{n}-\lambda_{n}\right) t}\left(1+s^{\prime}\right) \hat{A}(0)_{\mathrm{n}, \mathrm{n}^{\prime}}, \\
& \hat{A}_{2}(t)_{\mathrm{n}, \mathrm{n}^{\prime}}=\frac{1}{2} e^{i\left(s \omega_{n}+\lambda_{n}\right) t}\left(1-s^{\prime}\right) \hat{A}(0)_{\mathrm{n}, \mathrm{n}^{\prime}} .
\end{aligned}
$$

Thus the matrix element of $\hat{A}(t)_{\mathrm{n}, \mathrm{n}^{\prime}}=\hat{A}_{1}(t)_{\mathrm{n}, \mathrm{n}^{\prime}}+\hat{A}_{2}(t)_{\mathrm{n}, \mathrm{n}^{\prime}}$ is a sum of two terms, of which the first is nonzero for $s^{\prime}=+1$, while the second is nonzero for $s^{\prime}=-1$. One can show that the matrix elements given in Eqs. (73) and (74) are equal to the matrix elements of the Heisenberg operator $\hat{A}(t)_{\mathrm{n}, \mathrm{n}^{\prime}}=\left\langle\mathrm{n}\left|e^{i \Omega t} \hat{A}(0) e^{-i \Omega t}\right| \mathrm{n}^{\prime}\right\rangle$. For $\hat{A}^{+}(t)_{\mathrm{n}^{\prime}, \mathrm{n}}=\hat{A}_{1}^{+}(t)_{\mathrm{n}^{\prime}, \mathrm{n}}+\hat{A}_{2}^{+}(t)_{\mathrm{n}^{\prime}, \mathrm{n}}$ one obtains in a similar way

$$
\begin{aligned}
& \hat{A}_{1}^{+}(t)_{\mathrm{n}^{\prime}, \mathrm{n}}=\frac{1}{2} e^{i\left(+\lambda_{n}-s \omega_{n}\right) t}\left(1+s^{\prime}\right) \hat{A}^{+}(0)_{\mathrm{n}^{\prime}, \mathrm{n}} \\
& \hat{A}_{2}^{+}(t)_{\mathrm{n}^{\prime}, \mathrm{n}}=\frac{1}{2} e^{i\left(-\lambda_{n}-s \omega_{n}\right) t}\left(1-s^{\prime}\right) \hat{A}^{+}(0)_{\mathrm{n}^{\prime}, \mathrm{n}} .
\end{aligned}
$$

Formulas (73)-(76) describe the time evolution of the matrix elements of $\hat{A}(t)$ and $\hat{A}^{+}(t)$ calculated between two eigenstates of $\hat{\Omega}$. The frequencies appearing in the exponents are of the form $\pm \omega_{n+1} \pm \omega_{n}$. The intraband terms characterized by $\omega_{n}^{c}=\omega_{n+1}-\omega_{n}$ correspond to the cyclotron motion, while the interband terms characterized by $\omega_{n}^{Z}=\omega_{n+1}+\omega_{n}$ describe ZB. Different values of $s, s^{\prime}$ in the matrix elements of $\hat{A}_{1}(t)_{\mathrm{n}, \mathrm{n}^{\prime}}, \hat{A}_{2}(t)_{\mathrm{n}^{\prime}, \mathrm{n}}, \hat{A}_{1}^{+}(t)_{\mathrm{n}^{\prime}, \mathrm{n}}, \hat{A}_{2}^{+}(t)_{\mathrm{n}^{\prime}, \mathrm{n}}$ give contributions either to the cyclotron or to the $\mathrm{ZB}$ motion. The exact compact results given in Eqs. (73)-(76) indicate that our choice of $\hat{A}(t)$ and $\hat{A}^{+}(t)$ operators for the description of electrons in graphene in a magnetic field was appropriate.

To complete the operator considerations of ZB we calculate the matrix elements of $\hat{A}(t)$. We take $|\mathrm{n}\rangle=\left|n, k_{x}, 1\right\rangle$ and $\left|\mathrm{n}^{\prime}\right\rangle=\left|n+1, k_{x}, s\right\rangle$. The matrix elements of $\hat{A}(t)_{\mathrm{n}, \mathrm{n}^{\prime}}$ for the cyclotron and $\mathrm{ZB}$ components are

$$
\hat{A}(t)_{\mathrm{n}, \mathrm{n}^{\prime}}=(\sqrt{n+1} \pm \sqrt{n}) e^{i \omega(\sqrt{n} \mp \sqrt{n+1}) t},
$$

where the upper signs correspond to the cyclotron and the lower ones to the $\mathrm{ZB}$ motion, respectively. For $\hat{A}^{+}(t)_{\mathrm{n}, \mathrm{n}^{\prime}}$ there is

$$
\hat{A}^{+}(t)_{\mathrm{n}, \mathrm{n}^{\prime}}=(\sqrt{n+1} \pm \sqrt{n}) e^{i \omega(\sqrt{n+1} \mp \sqrt{n}) t} .
$$

The above results describe exact dynamics of electrons in monolayer graphene in the presence of an external magnetic field. This description is equivalent to the one given in the text but it is more general since it is not related to a specific electron wave packet.

\section{Appendix B}

We briefly discuss here the classical electron velocity and mass for a linear energy band of monolayer graphene, as they are often subjects of misunderstandings. Let us consider the conduction band and take $p \geq 0$, where the pseudo-momentum is $\boldsymbol{p}=\hbar \boldsymbol{k}$. Then the band 
dispersion is $E=u p$ and the classical velocity is

$$
v_{i}=\frac{\partial E}{\partial p_{i}}=\frac{d E}{d p} \frac{\partial p}{\partial p_{i}}=\frac{d E}{d p} \frac{p_{i}}{p}=\frac{d E}{d p} \frac{1}{p} \delta_{i j} p_{j},
$$

where $\delta_{i j}$ is the Kronecker delta function and we use the sum convention over the repeated index $j=1,2$. The electron mass tensor $\hat{m}$ relating the velocity to pseudo-momentum is defined by $\hat{m} \boldsymbol{v}=\boldsymbol{p}$. Then the inverse mass tensor $(1 \hat{/} m)$ is defined by

$$
v_{i}=\left(\frac{1}{m}\right)_{i j} p_{j} .
$$

Equating Eq. (79) with Eq. (80) we obtain

$$
\left(\frac{1}{m}\right)_{i j}=\frac{d E}{d p} \frac{1}{p} \delta_{i j}
$$

Thus the inverse mass tensor is a scalar: $1 / m=(d E / d p)(1 / p)$. Using the initial band dispersion one has $d E / d p=u$, so that $m=p / u=E / u^{2}$. This equality can be seen in two ways. First, it gives

$$
E=m u^{2},
$$

which is analogous to the Einstein relation between the particle energy and mass. Second, the formula

$$
m=\frac{E}{u^{2}}
$$

states that the mass vanishes at $E=0$ (or $p=0$ ), but is nonzero for $E>0$ (or $p>0$ ). These relations hold also for a more general "semi-relativistic" case of narrow-gap semiconductors, see Zawadzki (1997; 2006).

If a force $\boldsymbol{F}$ is applied to the electron, the relation

$$
\frac{d \boldsymbol{p}}{d t}=\boldsymbol{F}
$$

is always valid. In our case the pseudo-momentum $p=m u$, where $u$ is the constant velocity. Thus the force does not accelerate the electron, it only changes its mass.

One should add that, if one defined the mass by the relation of the force to acceleration: $\hat{M} \boldsymbol{a}=$ $\boldsymbol{F}$, the inverse mass would be given by the second derivative of the energy with respect to pseudo-momentum. For the linear band of graphene: $E=u p$, the second derivative of $E$ vanishes, so that such a mass would be infinitely large for all energies. Thus, it is not a useful quantity.

\section{References}

Ajiki, H. \& Ando, T. (1993). Magnetic Properties of Carbon Nanotubes. J. Phys. Soc. Jpn Vol. 62, 2470-2480

Barut, A. O. \& Bracken A. J. (1981). Zitterbewegung and the internal geometry of the electron. Phys. Rev. D Vol. 23, No. 10, 15 May 1981, 2454-2463 
Bjorken, J. D. \& Drell S. D. (1964). Relativistic Quantum Mechanics, McGraw-Hill, ISBN 9780072320022, New York

Bohm, D. (1952). Quantum Theory, Prentice-Hall, ISBN 0486659690, New York

Cannata, F.; Ferrari, L.; \& Russo, G. (1990). Dirac-like behaviour of a non-relativistic tight binding Hamiltonian in one dimension. Sol. St. Comun. Vol. 74, Issue 4, April 1990, 309-312

Castro Neto, A. H.; Guinea, F.; Peres, N. M. R.; Novoselov, K. S. \& Geim, A. K. (2009). The electronic properties of graphene. Rev. Mod. Phys. Vol. 81, No. 1, January-March 2009 2009, 109-162

Cserti, J. \& David, G. (2006). Unified description of Zitterbewegung for spintronic, graphene, and superconducting systems. Phys. Rev. B Vol. 74, No. 17, 15 November 2006, 172305 [4 pages] (arXiv:0604526)

Dreisow, F.; Heinrich, M.; Keil, R.; Tunnermann, A.; Nolte, S.; Longhi, S. \& Szameit, A. (2010). Classical Simulation of Relativistic Zitterbewegung in Photonic Lattices. Phys. Rev. Lett. Vol. 105, No. 14, 28 September 2010, 143902 [4 pages]

Englman, P. \& Vertesi, T. (2008). Large Berry phases in layered graphene. Phys. Rev. B Vol. 78, No. 20, 15 November 2008, 205311 [7 pages] (arXiv:0806.3726)

Feynmann, R. (1972). Statistical Mechanics; a set of lectures, W. A. Benjamin, ISBN 0201360764, Massachusetts

Gerritsma, R.; Kirchmair, G.; Zahringer, F.; Solano, E.; Blatt, R. \& Roos, C. F. (2010). Quantum simulation of the Dirac equation. Nature Vol. 463, 7 January 2010, 68-71 (arXiv:0909.0674)

Greiner, W. (1994). Relativistic Quantum Mechanics, Springer, ISBN 3540674578, Berlin

Jackson, J. D. (1975). Classical Electrodynamics, John Wiley \&. Sons, ISBN 9780471309321, New York

Jaynes, E. T. \& Cummings, F. W. (1963). Comparison of quantum and semiclassical radiation theories with application to the beam maser. Proc IEEE Vol. 51, No. 1, January 1963, 89-109

Johanning, M.; Varron, A. F. \& Wunderlich, C (2009). Quantum simulations with cold trapped ions. J. Phys. B Vol. 42, No. 15, 14 August 2009, 154009 [27 pages] (arXiv:0905.0118)

Katsnelson, M. I. (2006). Zitterbewegung, chirality, and minimal conductivity in graphene. Europ. Phys. J. B Vol. 51, No. 2, May 2006, 157-160 (arXiv:0512337)

Kronig, R. L. \& Penney, W. (1931). Quantum Mechanics of Electrons in Crystal Lattices. Proc. Roy. Soc. London Vol. 130, No. 814, 3 February 1931, 499-513

Krueckl, V. \& Kramer, T. (2009). Revivals of quantum wave packets in graphene. New J. Phys. Vol. 11, No. 9, September 2009, 093010 [22 pages]

Lamata, L.; Leon J.; Schatz, T. \& Solano, E. (2007). Dirac Equation and Quantum Relativistic Effects in a Single Trapped Ion. Phys. Rev. Lett. Vol. 98, No. 25, 22 June 2007, 253005 [4 pages] (arXiv:0701208)

Leibfried, D.; Blatt, R.; Monroe, C. \& Wineland, D. (2003). Quantum dynamics of single trapped ions. Rev. Mod. Phys. Vol. 75, No. 1, January-March 2003, 281-324

Lock, J. A. (1979). The Zitterbewegung of a free localized Dirac particle. Am. J. Phys. Vol. 47, No. 9, September 1979, 797-802

Lurie, D. \& Cremer, S. (1970). Zitterbewegung of quasiparticles in superconductors. Physica Vol. 50, No. 2, 30 November 1970, 224-240 
Maksimova, G. M, Demikhovskii, V Y \& Frolova, E V (2008). Wave packet dynamics in a monolayer graphene. Phys. Rev. B Vol. 78, No. 23, 15 December 2008, 235321 [7 pages] (arXiv:0809.0367)

Martinez, J. C.; Jalil, M. B. A. \& Tan, S. G. (2010). Klein tunneling and zitterbewegung and the formation of a polarized p-n junction in graphene. Appl. Phys. Lett. Vol. 97, No. 6, 9 Aug 2010, 062111 [3 pages](arXiv:0806.0222)

McCann, E. \& Fal'ko V. I. (2006). Landau-Level Degeneracy and Quantum Hall Effect in a Graphite Bilayer. Phys. Rev. Lett. Vol. 96, No. 8, 3 March 2006, 086805 [4 pages] (arXiv:0510237)

Novoselov, K. S.; Geim.; A. K.; Morozov, S. V.; Jiang, D.; Zhang, Y.; Dubonos, S. V.; Grigorieva, I. V. \& Firsov, A. A. (2004). Electric Field Effect in Atomically Thin Carbon Films. Science Vol. 306, No. 5696, 22 October 2004, 666-669 (arXiv:0410631)

Romera, E. \& de los Santos, F. (2009). Revivals, classical periodicity, and zitterbewegung of electron currents in monolayer graphene. Phys. Rev. B Vol. 80, No. 16, 15 October 2009, 165416 [5 pages] (arXiv:0911.4367)

Rusin, T. M. \& Zawadzki, W. (2007a). Zitterbewegung of nearly-free and tightly-bound electrons in semiconductors. J. Phys. Cond. Matter Vol. 19, No. 13, 4 April 2007, 136219 [18 pages] (arXiv:0605384)

Rusin, T. M. \& Zawadzki, W. (2007b). Transient Zitterbewegung of charge carriers in monoand bilayer graphene, and carbon nanotubes. Phys. Rev. B Vol. 76, No. 19, 15 November 2007, 195439 [7 pages] (arXiv:0702425)

Rusin, T. M. \& Zawadzki, W. (2008). Zitterbewegung of electrons in graphene in a magnetic field. Phys. Rev. B Vol. 78, No. 12, 15 September 2008, 125419 [9 pages] (arXiv:0712.3590)

Rusin, T. M. \& Zawadzki, W. (2009). Theory of electron Zitterbewegung in graphene probed by femtosecond laser pulses. Phys. Rev. B Vol. 80, No. 4, 15 July 2009, 045416 [9 pages] (arXiv:0812.4773)

Sakurai, J. J. (1987). Modern Quantum Mechanics, Addison-Wesley, ISBN 9780201539295, New York

Schliemann, J.; Loss, D. \& Westervelt, R. M. (2005). Zitterbewegung of Electronic Wave Packets in III-V Zinc-Blende Semiconductor Quantum Wells. Phys. Rev. Lett. Vol. 94, No. 20, 27 May 2005, 206801 [4 pages] (arXiv:0410321)

Schliemann, J. (2008). Cyclotron motion in graphene. New J. Phys. Vol. 10, April 2008, 034024 [9 pages] (arXiv:0802.1396)

Schrodinger, E. (1930). Ueber die kraeftefreie Bewegung in der relativistischen Quantenmechanik. Sitzungsber. Preuss. Akad. Wiss. Phys. Math. Kl. 24, 418-428. Schrodinger's derivation is reproduced in Barut \& Bracken (1981)

Semenoff, G. W. (1984). Condensed-Matter Simulation of a Three-Dimensional Anomaly. Phys. Rev. Lett. Vol. 53, No. 26, 24 December 1984, 2449-2452

Slonczewski, J. C. \& Weiss, P. R. (1958). Band Structure of Graphite. Phys. Rev. Vol. 109, No. 2, January 1958, 272-279

Smith, R. A. (1961). Wave Mechanics of Crystalline Solids, Chapman \& Hall, ISBN 0412093707, London

Vonsovskii, S. V.; Svirskii, M. S. \& Svirskaya, L. M. (1990). Zitterbewegung of a band electron. Teor. Matem. Fizika Vol. 85, No. 2, November, 1990, 211-221; Theor. and Math. Physics, Vol. 85, No. 2, 1159-1167 
Wallace, P. R. (1947). The Band Theory of Graphite. Phys. Rev. Vol. 71, No. 9, May 1947, 622-634 Wang, Y. X.; Yang, Z. \& Xiong, S. J. (2010). Study of Zitterbewegung in graphene bilayer with perpendicular magnetic field. Europhys. Lett. Vol. 89, No. 1, January 2010, 17007 [6 pages]

Winkler, R.; Zulicke, U. \& Bolte, J. (2007). Oscillatory multiband dynamics of free particles: The ubiquity of zitterbewegung effects. Phys. Rev. B Vol. 75, No. 20, 15 May 2007, 205314 [10 pages] (arXiv:0609005)

Zawadzki, W. (1997). Semirelativity in semiconductors. High Magnetic Fields in the Physics of Semiconductors II, Landwehr, G. \& Ossau. W, 755-768 World Scientific, ISBN 981-02-3990-4, Singapore

Zawadzki, W. (2005). Zitterbewegung and its effects on electrons in semiconductors. Phys. Rev. $B$ Vol. 72, No. 8, 15 August 2005, 085217 [4 pages] (arXiv:0411488)

Zawadzki, W. (2006). One-dimensional semirelativity for electrons in carbon nanotubes. Phys. Rev. B Vol. 74, No. 20, 15 November 2006, 205439 [4 pages] (arXiv:0510184)

Zawadzki, W. \& Rusin, T. M. (2008). Zitterbewegung (trembling motion) of electrons in narrow-gap semiconductors. J. Phys. Cond. Matter Vol. 20, No. 45, 12 November 2008, 454208 [5 pages] (arXiv:0805.0478)

Zawadzki, W. \& Rusin, T. M. (2010). Nature of electron Zitterbewegung in crystalline solids. Phys. Lett. A Vol. 374, No. 34, 26 July 2010, 3533-3537 (arXiv:0909.0463)

Zhang, X. \& Liu, Z. (2008). Extremal Transmission and Beating Effect of Acoustic Waves in Two-Dimensional Sonic Crystals. Phys. Rev. Lett. Vol. 101, No. 26, 31 December 2008, 264303 [4 pages] (arXiv:0804.1978) 


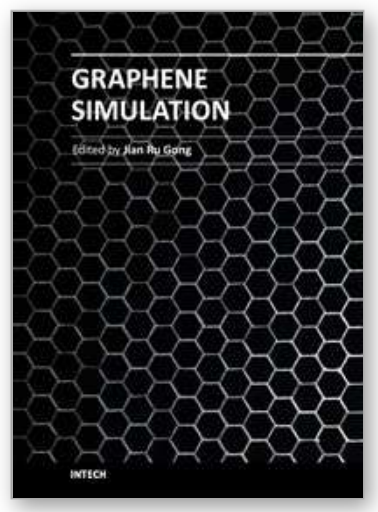

\author{
Graphene Simulation \\ Edited by Prof. Jian Gong
}

ISBN 978-953-307-556-3

Hard cover, 376 pages

Publisher InTech

Published online 01, August, 2011

Published in print edition August, 2011

Graphene, a conceptually new class of materials in condensed-matter physics, has been the interest of many theoretical studies due to the extraordinary thermal, mechanical and electrical properties for a long time. This book is a collection of the recent theoretical work on graphene from many experts, and will help readers to have a thorough and deep understanding in this fast developing field.

\title{
How to reference
}

In order to correctly reference this scholarly work, feel free to copy and paste the following:

Tomasz M. Rusin and Wlodek Zawadzki (2011). Zitterbewegung (Trembling Motion) of Electrons in Graphene, Graphene Simulation, Prof. Jian Gong (Ed.), ISBN: 978-953-307-556-3, InTech, Available from: http://www.intechopen.com/books/graphene-simulation/zitterbewegung-trembling-motion-of-electrons-ingraphene

\section{INTECH}

open science | open minds

\author{
InTech Europe \\ University Campus STeP Ri \\ Slavka Krautzeka 83/A \\ 51000 Rijeka, Croatia \\ Phone: +385 (51) 770447 \\ Fax: +385 (51) 686166 \\ www.intechopen.com
}

\author{
InTech China \\ Unit 405, Office Block, Hotel Equatorial Shanghai \\ No.65, Yan An Road (West), Shanghai, 200040, China \\ 中国上海市延安西路65号上海国际贵都大饭店办公楼405单元 \\ Phone: +86-21-62489820 \\ Fax: +86-21-62489821
}


(C) 2011 The Author(s). Licensee IntechOpen. This chapter is distributed under the terms of the Creative Commons Attribution-NonCommercialShareAlike-3.0 License, which permits use, distribution and reproduction for non-commercial purposes, provided the original is properly cited and derivative works building on this content are distributed under the same license. 